\title{
Efeito da ventilação não invasiva com pressão positiva contínua nas vias aéreas de pacientes oncológicos.
}

Dissertação apresentada à Faculdade de Medicina da Universidade de São Paulo para a obtenção do título de Mestre em Ciências.

Área de concentração: Fisiopatologia Experimental

Orientadora: Dra Elnara Márcia Negri

São Paulo 2008 
Dados Internacionais de Catalogação na Publicação (CIP)

Preparada pela Biblioteca da

Faculdade de Medicina da Universidade de São Paulo

Creprodução autorizada pelo autor

\section{Manfrim, Gabriela Marcon}

Efeito da ventilação não invasiva com pressão positiva contínua nas vias aéreas de pacientes oncológicos / Gabriela Marcon Manfrim. -- São Paulo, 2008.

Dissertação(mestrado)--Faculdade de Medicina da Universidade de São Paulo para obtenção do título de Mestre em Ciências.

Área de concentração: Fisiopatologia Experimental.

Orientadora: Elnara Márcia Negri.

Descritores: 1.Pressão positiva contínua nas vias aéreas 2.Muco 3. Síndrome do desconforto respiratório do adulto 4 . Dispnéia 5 . Cuidados paliativos 6 .Metástase neoplásica

USP/FM/SBD-192/08 
Aos meus queridos

Antonio e Dulcina, meus pais, e minha irmã Fabiana.

Pelo apoio e amor incondicionais

Ao meu amado Pedro.

Com quem compartilho vida, sonhos e vitórias. 


\section{AGRADECIMENTOS}

Aos meus pacientes e seus familiares, deste estudo e de toda minha vida profissional, pela confiança.

Às fisioterapeutas do Hospital A C Camargo, minhas primeiras colegas de profissão, com as quais muito aprendi, e que colaboraram direta ou indiretamente neste estudo, em especial a Carlinha, Lu Anitelli, Val Borba, Kátia, Luciana Titon, Eliana, Yone, Areta, Hellen, Lílian, Ju Mesti, Telma, Renata, Renata Francisco, Ana Cristina, Jaqueline, Karla Fabiana, Fernanda D’Almaso, Leonice, Daniela, Ana Carolina, Karin, Paula Regina, Adriana, enfim, a todas e todos os fisioterapeutas e estagiários do A C Camargo.

Às bibliotecárias do A C Camargo, em especial a Rose, por estar sempre disponível na etapa inicial deste trabalho, a Rita, Corina e Adriana do Hospital Sírio Libanês, por participarem da etapa final deste trabalho.

À equipe do Laboratório de Defesa Pulmonar porque sempre me senti parte desse time. Em especial a Regiani Oliveira, por preparar o palato de rã. Eu jamais teria conseguido sem você, Re.

Aos colegas da UTI, em especial o Dr Daniel Deheinzelin, médicos, enfermeiras e técnicos de enfermagem, por tornarem real o significado de atuação multiprofissional, em especial pela coleta de gasometria às enfermeiras Eliane, Daniela, Michele, Carol, Andréa, Suzana, e todas, enfim.

À minha amiga, madrinha, colega de turma e de mestrado, Flavia Cristina Almeida Leite Figueiredo, por ser sempre um exemplo. 
A toda minha família querida, por todo carinho e por entender minha ausência.

Às minhas "chefas" do A C Camargo, Silvia, Celena, Karin, Renata, Ana Paula e Soraia, pela primeira oportunidade profissional.

Meus ex-alunos de Taubaté e da Univap e Professores de Taubaté, inspiração para eu chegar até aqui.

À Paty Driusso, porque além de ser madrinha, amiga e incentivadora, entende de estatística como ninguém.

À Val Mira, behavioural scientist e amiga, por me ajudar a chegar até aqui.

À Dra Maria Teresa Cruz, pelo curso de psico-oncologia ter sido tão importante na minha formação profissional e pessoal e ajudar a definir o tema deste trabalho.

E por último, porque o melhor a gente sempre guarda pro final, agradeço imensamente à minha orientadora, Profa . Dra Elnara Márcia Negri por acreditar em mim, por ser além da minha orientadora, mãe da Bia e da Sofia, esposa do Toninho, médica do Sírio, amiga do Pepino, irmã da Ariadne, e por ser de longe a médica mais competente e dedicada que eu já conheci. Obrigada por respeitar meus limites e entender minhas inquietações acadêmicas, pessoais e espirituais. 
"Quanto mais você aprende, mais difíceis ficam as lições.

Quando aprendemos as lições, a dor se vai."

Elisabeth Kübler-Ross

"A RODA DA VIDA" 
Esta dissertação está de acordo com:

Referências: Adaptado de International Committee of Medical Journals Editors (Vancouver).

Universidade de São Paulo. Faculdade de Medicina. Serviço de Biblioteca e Documentação. Guia de apresentação de dissertações, teses e monografias. Elaborado por Anneliese Carneiro da Cunha, Maria Julia de A. L. Freddi, Maria F. Crestana, Marinalva de Souza Aragão, Suely Campos Cardoso, Valéria Vilhena. 2a ed. São Paulo: Serviço de Biblioteca e Documentação; 2005.

Abreviatura dos títulos dos periódicos de acordo com List of Journals Indexed in Index Medicus. 


\section{SUMÁRIO}

Lista de abreviaturas

Resumo

Summary

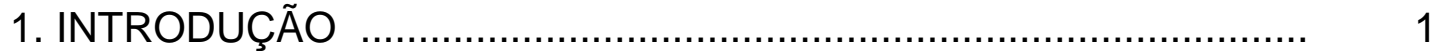

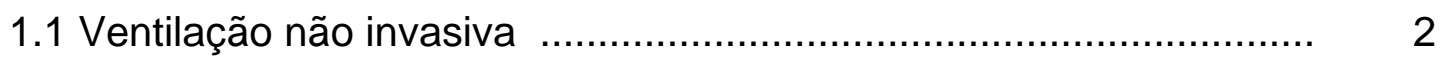

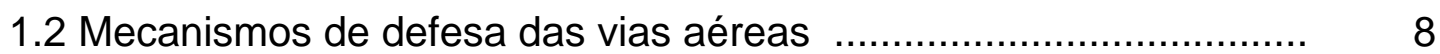

1.3 Insuficiência respiratória nos pacientes oncológicos $\ldots . . . \ldots \ldots \ldots \ldots \ldots . . . . . . .13$

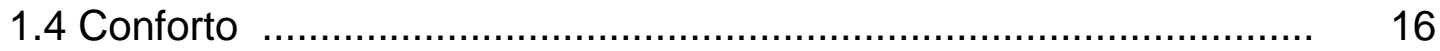

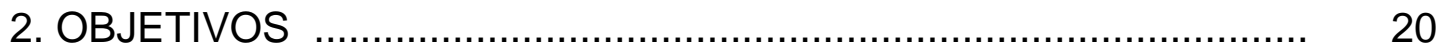

3. CASUÍSTICA E MÉTODO ......................................................... 22

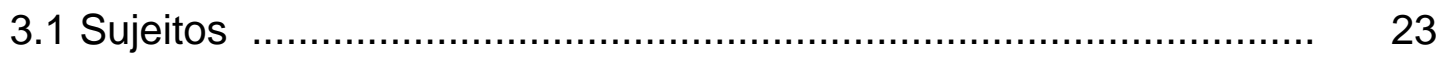

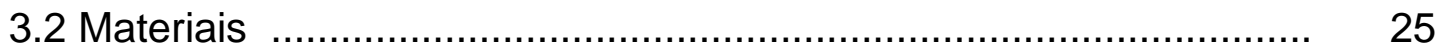

3.3 Desenho do estudo .......................................................... 26

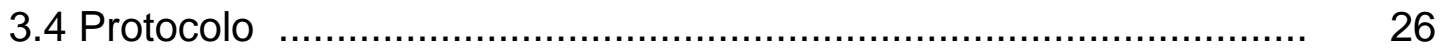

3.4.1 Fluxograma........................................................... 27

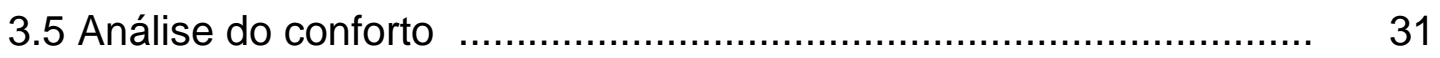

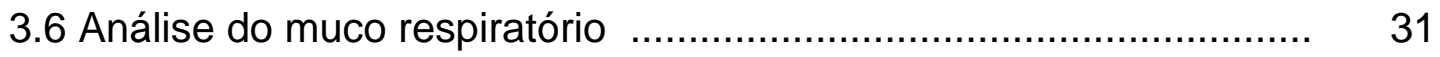

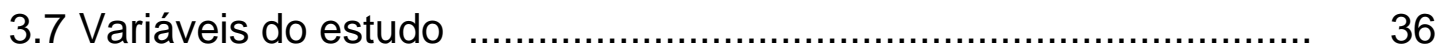

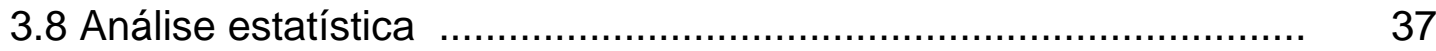

3.9 Questões éticas ............................................................ 38

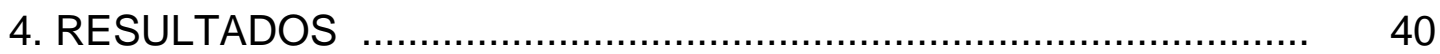

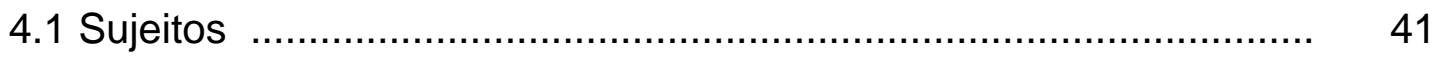


4.2 Causas da insuficiência respiratória......................................... 43

4.3 Propriedades físicas do muco ......................................... 44

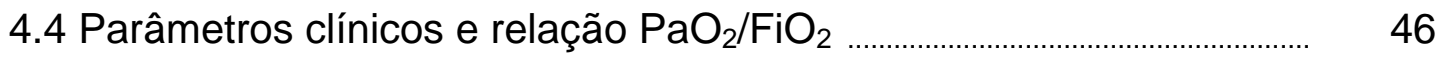

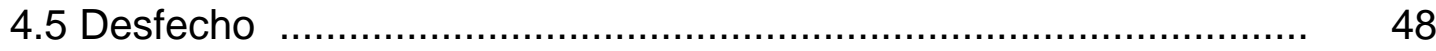

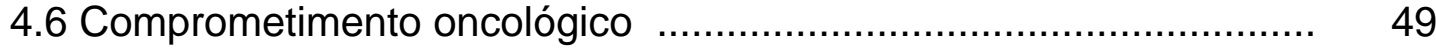

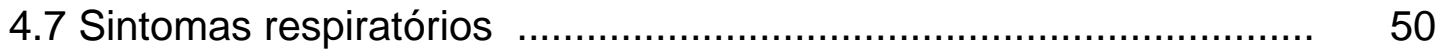

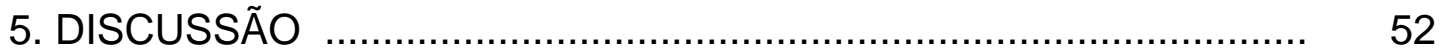

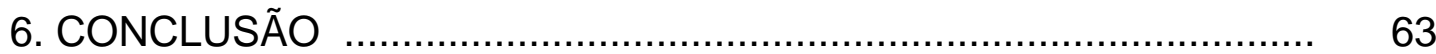

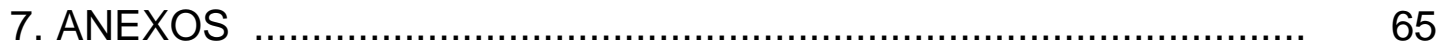

8. REFERÊNCIAS BIBLIOGRÁFICAS .......................................... 71 


\section{LISTA DE ABREVIATURAS}

\begin{tabular}{|c|c|}
\hline IRp & insuficiência respiratória \\
\hline IOT & intubação orotraqueal \\
\hline VNI & ventilação não ivasiva \\
\hline PEEP & $\begin{array}{l}\text { Pressão positiva ao final da } \\
\text { expiração }\end{array}$ \\
\hline CPAP & $\begin{array}{l}\text { pressão positiva contínua nas vias } \\
\text { aéreas }\end{array}$ \\
\hline SAOS & $\begin{array}{l}\text { Síndrome da apnéia obstrutiva do } \\
\text { sono }\end{array}$ \\
\hline DPOC & doença pulmonar obstrutiva crônica \\
\hline EPC & edema pulmonar cardiogênico \\
\hline $\mathrm{mg} / \mathrm{L}$ & miligramas por litro \\
\hline SDRA & $\begin{array}{l}\text { Síndrome do } \\
\text { respiratório agudo }\end{array}$ \\
\hline ICC & insuficiência cardíaca congestiva \\
\hline TMO & transplante de medula óssea \\
\hline f & freqüência respiratória \\
\hline DVA & drogas vasoativas \\
\hline FC & freqüência cardíaca \\
\hline PAS & pressão arterial sistólica \\
\hline PAD & pressão arterial diastólica \\
\hline $\mathrm{SaO}_{2}$ & saturação de oxigênio (gasometria) \\
\hline $\mathrm{SpO}_{2}$ & $\begin{array}{l}\text { saturação de oxigênio (oxímetro de } \\
\text { pulso) }\end{array}$ \\
\hline $\mathrm{cel} / \mathrm{mm}^{3}$ & $\begin{array}{l}\text { quantidade de células por milímetro } \\
\text { cúbico }\end{array}$ \\
\hline
\end{tabular}


SAPS II

TNM

Lpm

$\mu l$

HME

Vel rel índice de severidade da doença

sistema de estadiamento do tumor

litros por minuto

Microlitro

heat and moisture exchanger

Velocidade relativa 
Manfrim, G. M. Efeito da ventilação não invasiva com pressão positiva contínua nas vias aéreas de pacientes oncológicos. [dissertação]. São Paulo: Faculdade de Medicina, Universidade de São Paulo; 2007.

INTRODUÇÃO: A insuficiência respiratória acomete grande parte dos pacientes oncológicos levando a altos índices de mortalidade. A ventilação não invasiva ( $\mathrm{VNI}$ ) pode auxiliar seu manejo, mas seus efeitos ainda são pouco conhecidos sobre os mecanismos de defesa pulmonar. OBJETIVOS: Observar o efeito da VNI com máscara facial usando-se geradores de fluxo com pressão positiva contínua (CPAP) e ventilador microprocessado no modo pressão de suporte + pressão positiva ao final da expiração (PSV + PEEP), a fim de verificar impacto nas propriedades viscoelásticas do muco respiratório e o conforto proporcionado ao paciente. MÉTODOS: A VNI foi instalada após diagnóstico de insuficiência respiratória em dezenove pacientes, admitidos nas unidades de tratamento intensivo do Hospital A. C. Camargo, sendo nove submetidos ao CPAP e dez com PSV + PEEP. Foram colhidos antes e após uma hora de VNI: os dados clínicos, secreção nasal, gasometria, e o grau de conforto através de uma escala visual. As propriedades físicas do muco (transportabilidade in vitro, adesividade e wettabilidade ou hidrofobicidade) foram avaliadas respectivamente no palato de rã, máquina da tosse e ângulo de contato. RESULTADOS: Os grupos eram homogêneos entre si em relação à idade, sexo, tipo e estadiamento do tumor e SAPS II. Em relação às propriedades físicas do muco, houve um aumento da transportabilidade in vitro do muco nasal com o sistema PSV + PEEP $(p=0,04)$ e um aumento na wettabilidade no grupo CPAP $(p=0,06)$. Os dois sistemas foram eficazes em melhorar significativamente os sinais vitais, a $\mathrm{PaO}_{2} / \mathrm{FiO}_{2}$, o padrão e o conforto respiratório e em evitar a intubação traqueal nas primeiras 24 horas $(p<0,05)$. Entretanto, independentemente do tipo de sistema de VNI usado, foram encontrados altos índices de intubação endotraqueal e mortalidade no seguimento destes pacientes. CONCLUSÃO: As propriedades físicas do muco (transportabilidade in vitro e wettabilidade) se alteraram após uma hora de uso da VNI e parecem ser dependentes da temperatura e umidificação dentro da máscara. A VNI mostrou-se útil em reverter a insuficiência respiratória em pacientes selecionados, ou pelo menos em trazer conforto para pacientes hipoxêmicos que a princípio recusam a intubação endotraqueal.

Descritores: pressão positiva contínua nas vias aéreas, muco, síndrome do desconforto respiratório agudo, dispnéia, cuidados paliativos, metástase neoplásica. 
Manfrim, G. M. Effects of noninvasive ventilation with continuous positive pressure on the airways of oncologic patients. [dissertation]. São Paulo: Faculdade de Medicina, Universidade de São Paulo; 2007.

INTRODUCTION: Respiratory failure is a common situation among cancer patients leading to high rates of mortality. Noninvasive ventilation (NIV) can help its management, but its effects are still unknown regarding the pulmonary defense mechanisms. OBJECTIVES: Observe the effect of NIV with facial mask using a flow generator with continuous positive pressure (CPAP) and standard intensive care unit ventilator using pressure support ventilation + positive end expiratory pressure (PSV + PEEP), to verify impact on the physical properties of respiratory mucus and the comfort provided to the patient. METHODS: NIV was started after diagnosis of respiratory failure in nineteen patients, admitted in the intensive care unit of the A. C. Camargo Hospital. Nine patients were submitted to CPAP and ten to PSV + PEEP. Nasal mucus, blood gases, and the degree of comfort through a visual scale were accessed before and after one hour. The physical properties of nasal mucus (transportabilility in vitro, adhesivity and wettability or hydrofobicity) were evaluated respectively by frog palate, cough machine and contact angle. RESULTS: Groups had similar characteristics about age, sex, tumor and SAPS II score. Regarding the physical properties of the mucus, there was an increase in mucus transportability (by the frog palate model) with the system PSV + PEEP $(p=0.04)$ and an increase in the contact angle in the CPAP groupo $(p=0.06)$. The two systems were effective in improving the vital signs, the $\mathrm{PaO} 2 / \mathrm{FiO} 2$, the respiratory pattern and comfort and avoiding endotracheal intubation in the first 24 hours $(p<0.05)$. However, regardless of the type of NIV system used, high rates of endotracheal intubation and mortality were found. CONCLUSION: The physical properties of the mucus (transportability in vitro and wettability) changed after an hour of use of the NIV as a result of temperature and humidification into the mask. NIV was useful in reversing the respiratory failure in selected patients, or at least in bringing comfort for those who refuse endotracheal intubation.

Descriptors: continuous positive pressure, mucus, acute respiratory distress syndrome, palliative care, metastatic cancer. 
INTRODUÇÃO 


\section{1- INTRODUÇÃO}

Insuficiência respiratória (IRp) é um evento comum nos pacientes oncológicos em algum momento da doença, seja pelo comprometimento pulmonar ou mesmo decorrente do tratamento. O prognóstico é pior quando estes pacientes necessitam de intubação orotraqueal (IOT) e nesse contexto a ventilação mecânica não invasiva (VNI) vem se mostrando como ótima alternativa em grupos selecionados de pacientes como os imunodeprimidos e com causas reversíveis de IRp (Nava e Cuomo, 2004).

Este estudo aborda o impacto da VNI no paciente oncológico crítico através de máscara facial, com dois tipos de sistemas: um gerador de fluxo e um aparelho de ventilação mecânica não invasiva.

Como não há dados na literatura sobre o efeito da VNI no sistema mucociliar destes pacientes, o objetivo deste estudo é verificar o efeito da pressão positiva contínua sobre os mecanismos de defesa pulmonar, pela análise do muco respiratório no palato de rã, na máquina da tosse e pelo ângulo de contato.

A reversão da insuficiência respiratória e o conforto proporcionado pela VNI também são avaliados, a fim de trazer as melhores evidências no tratamento da insuficiência respiratória neste grupo especial de pacientes.

\subsection{VENTILAÇÃO NÃO INVASIVA (VNI):}

De acordo com o Consenso de Ventilação Mecânica (2007), a ventilação mecânica não invasiva com pressão positiva (que neste trabalho 
será chamada apenas de VNI) é definida como uma técnica de ventilação mecânica onde o suporte ventilatório é oferecido através de máscaras nasais, oronasais (mais conhecidas como máscaras faciais), além das mais recentes máscaras faciais totais e do tipo capacete. A máscara facial é a mais usada no tratamento da IRp por permitir a correção mais rápida nas trocas gasosas.

Usualmente refere-se à VNI como a oferta de uma pressão de suporte inspiratória associada a uma pressão positiva ao final da expiração (PEEP) por máscara nasal ou facial. Apesar da pressão positiva contínua nas vias aéreas (CPAP) não assistir à inspiração e não ser um modo ventilatório verdadeiro poderá ser considerado como uma forma de VNI quando usada como terapia para tratar IRp (Hill et al., 2007).

Os objetivos principais da ventilação mecânica não invasiva são os mesmos da ventilação mecânica invasiva (VMI), mas sem o uso do tubo traqueal:

- Manutenção das tocas gasosas pulmonares (correção da hipoxemia e garantia da ventilação alveolar para eliminação do gás carbônico $\left.\mathrm{CO}_{2}\right)$

- Diminuição do trabalho respiratório (prevenção ou tratamento da fadiga muscular);

- Manutenção dos volumes pulmonares (prevenção ou correção do colapso alveolar);

- Diminuição da dispnéia (conforto). 
Num estudo de revisão, Mehta e Hill (2001), demonstraram que o interesse pela VNI vem aumentando a partir da década de 90 a fim de se evitar as complicações da ventilação invasiva devido à intubação traqueal. Complicações estas bem definidas em três categorias principais:

1- Diretamente relacionadas à intubação e à ventilação mecânica: hipotensão, arritmias, lesões de laringe, traquéia, esôfago e vasos, hemorragias, falso trajeto, traumas diretos nos dentes, aspiração de conteúdo gástrico, mediastinites;

2- Aquelas causadas pela perda dos mecanismos de defesa das vias aéreas, (o tubo traqueal como conduto direto para as vias aéreas inferiores de contaminações externas), facilitando a ocorrência de pneumonia nosocomial, inflamações, sinusites, prejuízo às funções do epitélio ciliado das vias aéreas superiores, aumento da produção de muco;

3- Aquelas que ocorrem após a retirada da prótese endotraqueal: hemoptise, tosse ineficaz, obstrução alta por disfunção das cordas vocais ou estenose de traquéia.

Neste mesmo estudo, os autores afirmam que as complicações da ventilação mecânica invasiva só serão evitadas com a VNI, se os candidatos forem cuidadosamente selecionados, usando-se normas bem estabelecidas de inclusão.

Assim, a seleção criteriosa dos pacientes é fundamental para o sucesso da VNI e segundo Schettino et al. (2000), deve-se ter pelo menos dois dos critérios a seguir: 
- Desconforto respiratório com dispnéia moderada a severa, uso de musculatura acessória e ou respiração paradoxal;

- $\mathrm{pH}<7,35$ e $\mathrm{PaCO}_{2}>45 \mathrm{mmHg}$;

- Freqüência respiratória (f) > 25 rpm (adultos).

As contra indicações absolutas da VNI são:

- Parada cardiorrespiratória;

- Instabilidade cardiovascular (choque, arritmias severas, infarto agudo do miocárdio);

- Paciente não colaborativo;

- Pós-operatório de cirurgia facial;

- Risco de aspiração e dificuldade de manipulação de secreções;

- Incapacidade de manter vias aéreas pérveas.

Contra indicações relativas:

- Pós-operatório de cirurgia esofágica e ou gástrica recente;

- Secreção abundante;

- Síndrome da angústia respiratória aguda com hipoxemia grave. Quanto aos benefícios da VNI têm-se:

- A manutenção das vias aéreas intactas;

- Preservação dos mecanismos de defesa das mesmas;

- Permitir que o paciente possa verbalizar, alimentar-se e expectorar secreções;

- Pode ainda reduzir os riscos de infecção nosocomial e sinusite;

- Também produz mais conforto e menos custos ao hospital se comparado com os custos da IOT; 
A VNI pode ainda ser administrada fora das unidades de terapia intensiva (UTIs), desde que haja adequada assistência de enfermagem e fisioterapia, com treinamento da equipe no uso da $\mathrm{VNI}$, racionalizando melhor os leitos das unidades críticas (Mehta e Hill, 2001).

Ainda neste estudo os efeitos negativos freqüentemente encontrados na VNI são:

- Dor do osso nasal, inclusive com eritema e ulceração da pele causada pela pressão da máscara na região. Isto pode ser minimizado diminuindo a pressão da máscara e aplicando placas de pele artificial ou protetores nasais de silicone nos pontos de maior pressão ou com o uso das máscaras faciais totais;

- Ressecamento das mucosas causado pelos altos fluxos de ar seco diretamente na boca e nariz;

- Distensão gástrica;

- Os vazamentos ao redor da máscara que podem causar úlceras de córnea (resolvidas com gel lubrificante ocular antes da administração da VNI).

Os maiores sucessos do uso da VNI são na doença pulmonar obstrutiva crônica (DPOC) exacerbada, nas doenças neuromusculares em estágio crônico e no edema pulmonar cardiogênico (EPC) (Schettino et al., 2000; Consenso de VM, 2007), além de evitar a IOT em pacientes imunodeprimidos e no desmame de pacientes com DPOC sob ventilação invasiva (Hill et al., 2007). 
A falência da VNI tem sido registrada em $7 \%$ a $42 \%$ dos pacientes (Metha e Hill, 2001).

Segundo a literatura, se após uma hora de tratamento não houver melhora do quadro, raramente o paciente vai se beneficiar da VNI e quanto mais se postergar a IOT, maiores as complicações. A equipe deve sempre estar perto do paciente para encorajar, controlar vazamentos, e verificar se há necessidade de sedativos e também deve estar alerta para verificar sinais de intolerância ao procedimento ou se há piora das condições clínicas (Antonelli et al., 2001; Mehta e Hill, 2001;).

As causas de falência incluem:

- Falha na melhora da ventilação alveolar;

- Intolerância relacionada à máscara (sensação de claustrofobia e de alta pressão do ar);

- Desconforto em coordenar a respiração com o ventilador (geralmente isto aparece quando se usa aparelho de VMI como VNI, com as válvulas de demanda muito duras, menos sensíveis ao esforço do paciente);

- Parâmetros ventilatórios inadequadas, obstrução nasal, retenção de secreções e escape de ar excessivo.

Um cuidado muitas vezes esquecido na administração da VNI é o condicionamento do ar inspirado. Em pacientes intubados, o tubo endotraqueal suprime a capacidade de aquecimento, umidificação e de defesa das vias aéreas. Aquecedores elétricos ou o nariz artificial são prontamente instalados após a IOT, mas a umidificação e o aquecimento do 
ar inspirado na VNI é relegada a um segundo plano, pela idéia difundida na literatura de que as vias aéreas continuam preservadas.

Os sintomas respiratórios causados pela inalação dos altos fluxos de ar frio e seco são apontados como importante fator de falha no tratamento com VNI em pacientes com SAOS. O ar frio e seco do CPAP nasal causa uma liberação local de mediadores inflamatórios representando uma resposta aguda de defesa contra a agressão nas vias aéreas superiores (Oliveira, 2006). Abandono do tratamento pode ser comum devido esse fator. Mesmo em indivíduos saudáveis, ressecamento nasal e de garganta são freqüentes quando recebem VNI com ar frio e sem umidificação e com vazamento pela boca (Oliveira, 2006).

Hill et al. em seu estudo de revisão de 2007, mostram as melhores evidências sobre o uso da VNI nas diferentes causas de IRp através da seleção criteriosa dos pacientes e das melhores técnicas empregadas. Mas não existem dados sobre o efeito da VNI nos mecanismos de defesa das vias aéreas de pacientes oncológicos com insuficiência respiratória, cuja intubação traqueal é evitada principalmente nos pacientes imunossuprimidos e nos pacientes terminais que recusam a IOT.

\subsection{MECANISMOS DE DEFESA DAS VIAS AÉREAS}

O trato respiratório é recoberto por tecido pseudoestratificado colunar ciliado que reveste desde porções médias das cavidades nasais até 
bronquíolos terminiais. Esta porção é chamada de vias aéreas de condução (Saldiva, 1990; Lorenzi e Saldiva, 1991).

A função das vias aéreas de condução é proteger e condicionar o ar inspirado até este chegar aos alvéolos em condições ideais onde então serão efetuadas as trocas gasosas, sendo esta a principal função do sistema respiratório (Saldiva, 1990; Lorenzi e Saldiva, 1991).

As vias aéreas de condução são recobertas por uma camada de muco (produzido pelas células secretoras: células mucosas, serosas, e da Clara). O muco respiratório é dividido em duas camadas: camada gel, superior, de material viscoelástico e a camada sol (ou periciliar), inferior, composta de um fluido seroso (Saldiva, 1990).

Os cílios são prolongamentos citoplasmáticos das células ciliadas (figura1) e a freqüência de batimento ciliar é de 17 a 25 batimentos/segundo. O transporte do muco é feito no sentido cranial, em direção à orofaringe, onde é deglutido ou expectorado (Willians et al., 1996).

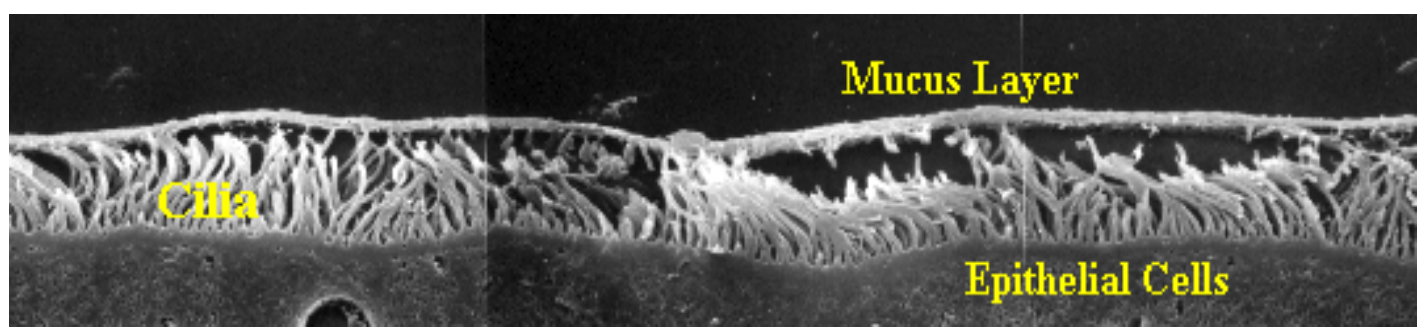

Figura1: Eletromiografia de varredura mostrando a interface mucociliar: www.users.umassmed.edu/michael.sanderson/mjslab/Cilia_Page.htm

O clearance mucociliar nasal é o primeiro mecanismo de defesa pulmonar e sua eficiência depende de quatro fatores principalmente: 
magnitude do batimento ciliar, quantidade de muco, acoplamento do muco na camada periciliar e das propriedades físicas do muco (Oliveira, 2005).

Segundo Yagi (2002), a falência do transporte mucociliar:

- Facilita a colonização de bactérias, aumentando os riscos de infecção respiratória;

- Aumenta o tempo de contato de agentes agressores com o epitélio respiratório (hipótese da associação da falência do transporte mucociliar com a carcinogênese pulmonar proposto por Zayas et al., 1990);

- Causa estase de muco acarretando graves distúrbios na ventilação, com aumento da resistência ao fluxo aéreo.

Processos irritativos repetidos na árvore respiratória alteram seu epitélio e conseqüentemente a composição do muco. As células ciliadas diminuem em número e há aumento das células caliciformes e de secreção mucosa. Surge um muco mais viscoso, que ficará mais tempo retido no sistema respiratório (Oliveira, 2005).

As elevações na temperatura ambiente também aumentam a freqüência de batimento ciliar, assim como evaporação e condensação excessivas alteram as propriedades viscoelásticas do muco alterando também a freqüência de batimento ciliar (Williams et al., 1996).

No levantamento bibliográfico realizado, foram encontrados estudos relacionando os efeitos do CPAP nasal na transportabilidade do muco nasal ou no clearance mucociliar nasal. A população estudada era de sujeitos saudáveis (Oliveira et al., 2006) ou com sinusite (Constantinidis et al., 2000) 
ou ainda com outras doenças das vias aéreas superiores e inferiores como a alergia nasal e a asma (Ogino et al.,1993). Estes estudos foram feitos através do teste da sacarina e apenas o estudo de Oliveira (2006) correlaciona o tempo de trânsito da sacarina nasal com a alteração do transporte do muco no palato de rã.

Não existem estudos sobre o efeito da ventilação mecânica não invasiva com pressão positiva em pacientes com IRp ou mesmo em pacientes oncológicos, apesar de todos pelo menos mencionarem a importância da VNI em preservar os mecanismos de defesa das vias aéreas em relação a intubação orotraqueal (Nava e Cuomo, 2004; Consenso de VM, 2000).

Um ponto importante a ser mencionado é o efeito, estudado por Sayit et al. (2000), da quimioterapia nos mecanismos de defesa das vias aéreas em pacientes recebendo mitomicina $\mathrm{C}$ e isofosfamida e doxorubicin, os três agentes reconhecidamente pneumotóxicos. Neste estudo, foi verificado o clearance de um radioisótopo inalado através de imagens radiológicas seriadas do pulmão antes e após um ciclo de quimioterapia.

Os autores encontraram uma diminuição no clearance do isótopo inalado tanto em regiões centrais como periféricas do pulmão, sugerindo como efeito agudo da quimioterapia a toxicidade no sistema mucociliar, antes de ocorrer alteração na permeabilidade da membrana alvéolo-capilar.

Surico et al. (2001), observou que em pacientes com até 17 anos curados de tumores de cabeça e pescoço, e submetidos à radioterapia de crânio, tinham mais infecções de vias aéreas superiores e diminuição da 
transportabilidade do muco nasal verificado pelo teste da sacarina e que esta resposta estava relacionada com a dose de radiação recebida.

Uma das grandes dificuldades no estudo das propriedades físicas do muco respiratório é a ausência de um método simples e não invasivo para a coleta do muco em quantidade suficiente para serem avaliadas repetidamente ou mesmo numa única ocasião.

Diversos métodos têm sido empregados em condições normais tanto em animais quanto em humanos (Yagi, 2002), mas nada foi descrito em pacientes oncológicos ou em pacientes com insuficiência respiratória respirando espontaneamente.

Sabe-se que os pacientes criticamente doentes mesmo sem manipulação das vias aéreas já apresentam comprometimento do seu transporte mucociliar e este seria um mecanismo potencial para o desenvolvimento de infecções nosocomiais e do aumento da morbidade e mortalidade destes pacientes (Nakagawa et al., 2005).

Infelizmente pacientes críticos constantemente recebem oxigenioterapia, aspirações nasotraqueais, IOT, VNI, drogas depressoras do sistema nervoso central, altos volumes de soro fisiológico e eletrólitos, hipotermia no centro cirúrgico, quimioterapia, radioterapia torácica e todos estes fatores podem prejudicar drasticamente 0 transporte mucociliar (Nakagawa et al., 2005).

A medida deste prejuízo e a busca de soluções que minimizem os efeitos deletérios do tratamento intensivo devem nortear as pesquisas clínicas. O estudo das propriedades físicas do muco pode trazer as 
evidências necessárias para o maior sucesso do tratamento de pacientes críticos com a ventilação não invasiva.

\subsection{INSUFICIÊNCIA RESPIRATÓRIA NOS PACIENTES}

\section{ONCOLÓGICOS}

Câncer é um termo genérico usado para descrever mais de 200 doenças individuais, que progridem de maneiras diferentes, mas têm como característica comum o crescimento de células anormais de qualquer tecido do hospedeiro (Love, 1999).

Estas células proliferam-se localmente, invadem e atravessam barreiras tissulares normais, reproduzindo-se indefinidamente. As massas de células anormais disseminam-se pelo organismo (processo metastático) e levam o hospedeiro à morte se não forem erradicadas (Love, 1999).

Os pulmões sofrem disseminação linfangítica de vários tumores sólidos: carcinoma mamário, de pulmão, de próstata, de pâncreas e estômago (Love,1999).

Segundo Nava e Cuomo (2004), infecções, hemorragias, síndrome do extravasamento capilar, toxicidade pela radioterapia ou à quimioterapia também transformam o pulmão em órgão alvo destas complicações.

Lesão primária do endotélio vascular ou lesão das células epiteliais alveolares com lesão microvascular secundária podem ocorrer bem como extravasamento de líquidos e proteínas, primeiro para o espaço intersticial e nos casos mais graves para os alvéolos. Se sua localização for difusa, o 
edema alveolar é um importante fator contribuinte para síndrome do desconforto respiratório agudo (SDRA) (Robbins et al.,1996).

Além disso, muitos pacientes oncológicos apresentam comorbidades como história de tabagismo, doença pulmonar obstrutiva crônica (DPOC) e insuficiência cardíaca congestiva (ICC), que quando exacerbadas podem desencadear quadros de IRp bastante comuns, mesmo sem o acometimento pulmonar pelo câncer (Nava e Cuomo, 2004).

A síndrome da hemorragia alveolar difusa está relacionada com uma série de doenças, principalmente nos pacientes imunodeprimidos (Schwarz, 2001). O que caracteriza esta síndrome é o sangramento dentro dos espaços alveolares, mas a hemoptise nem sempre está presente, mesmo quando na sua forma mais severa. Freqüentemente estes pacientes necessitam de suporte ventilatório.

Em 1985, Luedke et al. relataram um estudo de caso de pacientes que desenvolveram insuficiência respiratória aguda com ou sem progressivo infiltrado intersticial, quase sempre fatal, com queda progressiva da $\mathrm{PaO}_{2}$ após administração de mitomicina C e vindesina (vinca alcalóide), ambos agentes quimioterápicos usados no tratamento de tumores sólidos. Nenhum dos pacientes do estudo acima tinha febre, descompensação cardíaca, nem leucocitose ou leucopenia. Já no estudo de Azoulay et al., (2001), quase $40 \%$ dos pacientes eram neutropênicos, e no trabalho de Hilbert et al. (2001) toda a população estudada era de pacientes neutropênicos e febris.

Sabe-se que o prognóstico de pacientes oncológicos com IRp que necessitam de VM é ruim, mas a mortalidade varia com o diagnóstico de 
base: $95 \%$ dos pacientes com transplante de medula óssea (TMO) vão a óbito; $75 \%$ a $90 \%$ para doenças malignas hematológicas; e $70 \%$ a $90 \%$ dos pacientes com tumores sólidos vão a óbito (Pastores, 2001).

Em outros modelos, câncer metastático, TMO, leucemia aguda, linfoma, arritmias cardíacas, e hipotensão são preditivos de aumento na mortalidade intra-hospitalar em unidade de tratamento intensivo (UTI) (Groeger et al, 1999).

Brandão, em 2003, mostrou que dos pacientes oncológicos admitidos em uma UTI especializada, $24 \%$ são admitidos recebendo tratamento efetivo ou pleno para o seu tumor, mas evoluem recebendo tratamento paliativo e destes, $10 \%$ vão a óbito quanto maior o tempo de permanência na UTI. $73 \%$ dos pacientes que foram a óbito neste estudo tinham dispnéia.

Os pacientes oncológicos evoluem com IRp pela própria queda do estado geral, por depleção protéica e fadiga crônica ou por agudização de doenças crônicas como DPOC e ICC e podem não receber IOT ou a VNI simplesmente por serem terminais, lembrando que o alívio da dispnéia é um dos objetivos a serem alcançados em cuidados paliativos.

Em pacientes sem indicação de IOT, a VNI muitas vezes não é oferecida, ou é utilizada como alternativa à sedação contínua. Faltam estudos para definir melhor qual subgrupo destes pacientes terminais se beneficiaria com a VNI para garantir conforto e respeitar o desejo do paciente que recusa sofrimento inútil em prolongar a vida artificialmente com a IOT (Schettino et al., 2005). 


\subsection{CONFORTO}

Poucos trabalhos estudaram o grau de conforto proporcionado aos pacientes submetidos a VNI, mesmo esta questão sendo levantada em vários estudos.

Sempre existe um grupo de pacientes que não tolera a terapia com VNI. Exclusivamente o estudo de Gregoretti et al. (2002), registrou o conforto oferecido por dois tipos de máscara facial de VNI, através da escala modificada de Calderini et al. (1999)* com 5 graus de melhora do conforto: 1- muito pouco; 2- pouco; 3- suficiente; 4- bom; 5- muito bom. Uma limitação relatada pelos autores deste estudo foi não conseguir excluir o conforto proporcionado só pela máscara, mas a medida foi dada pela composição entre o tipo de máscara e os diferentes modos ventilatórios usados neste estudo.

O estudo de Oliveira (2005) registrava os escores de sintomas respiratórios mensurados em centímetros.

Delelaux et al. (2001) perguntava aos pacientes após uma hora de terapia o efeito sobre sua dispnéia que podia variar de +2 até -2 numa escala analógico-visual. Este trabalho comparava o tratamento com oxigenioterapia versus $\mathrm{o}$ tratamento com CPAP e oxigenioterapia associados, e mostrou que apesar dos efeitos benéficos do CPAP este não foi capaz de evitar a IOT em pacientes com IRp aguda, hipoxemia severa e sem hipercapnia.

\footnotetext{
${ }^{*}$ Calderinie et al. Timed-cycled versus flow-cycled noninvasive pressure support ventilation. Intensive Care Med. 1999; 25: 662-7.
} 
Na maioria destes estudos os autores são contra o uso de sedativos e não há menção se havia pacientes que já faziam uso de benzodiazepínicos ou antidepressivos, que poderiam tolerar melhor a terapia se devidamente medicados e assim contribuir com o sucesso do seu tratamento.

Além disso, a falta de ar, entre outros sintomas vegetativos, pode ser decorrente tanto da patologia orgânica quanto mental, confundindo o diagnóstico. Em um hospital, torna-se difícil diferenciar casos psiquiátricos, quando se combinam, além do sofrimento psíquico, doenças físicas e problemas sociais (Botega et al., 1995).

Ferramentas que não exijam do paciente esforço para preencher questionários ou submetê-los a extensos interrogatórios e que sejam de fácil visualização e entendimento devem ser usadas para medir o grau de desconforto respiratório e a eficácia da intervenção terapêutica (Pompilio, 2000).

Existem muitos estudos sobre o uso da máscara facial com pressão positiva contínua em vias aéreas em pacientes com insuficiência respiratória aguda tanto de origem cardíaca quanto pulmonar (Bernsten et al., 1991; Meduri et al., 1994, 1996; Brochard et al., 1995; Ambrosino, 1996; Scarpinella-Bueno et al., 1997; Delelaux et al., 2000; Antonelli et al., 1998, 2000, 2001; Brochard, 2002), e sobre pacientes oncológicos, a maioria em pacientes neutropênicos e imunocomprometidos (Mehta e Hill, 2001; Hilbert et al., 2001).

A VNI está começando a ser estudada em pacientes terminais onde não há indicação de intubação traqueal com risco de prolongar 
dolorosamente a vida, e vem se mostrando útil em trazer conforto para pacientes hipoxêmicos que recusam a IOT (Shee et al., 2003; Meert et al., 2003; Levy et al., 2004; Nava e Cuomo, 2004; Cuomo, 2004).

É importante ressaltar que na grande parte dos trabalhos de ventilação não invasiva os sistemas usados são aparelhos de ventilação invasiva adaptados às máscaras faciais, como o Puritan Bennett 7200 (Puritan Bennett, USA), Servo 900 C e o Servo 300 Siemens (Siemens Elema, Uppsala, Sweden) ou ainda aparelhos desenvolvidos exclusivamente para esse fim como o Bipap Vision Ventilator (Respironics, USA) (Brochard et al., 1995; Guérin et al., 1997; Antonelli et al., 1998; Varon et al., 1998; Conti et al., 1998; Antonelli et al., 2000; Hilbert et al., 2001; Azoulay et al., 2001; Antonelli et al., 2001).

Poucos trabalhos fazem uso do sistema de geradores de fluxo como o Vital Flow 100 CPAP Flow Generator (Bersten et al., 1991; Delelaux et al., 2000).

O uso dos aparelhos de ventilação mecânica invasiva quando usados sob ventilação não invasiva podem apresentar alguns problemas como a deficiente sincronia ventilador-paciente. Esta assincronia pode causar autociclagem, vazamentos e altas pressões, o que geralmente resulta em importante desconforto ao paciente. Isto foi bem demonstrado em um trabalho feito com simuladores mecânicos por Fu (2000).

Vale ressaltar que o gerador de fluxo tem um custo significativamente mais baixo se comparado com os aparelhos de ventilação mecânica microprocessados. Além de serem de fácil manuseio e transporte, (o que 
permite seu uso fora das UTls e unidades fechadas), garantem pronto atendimento da IRp em situações reversíveis como o EPC e o broncoespasmo, já que seu uso está bem demonstrado nestas situações, (Brochard et al., 1995; Meduri, 1996), mas ainda não há trabalhos demonstrando seu efeito em pacientes oncológicos criticamente doentes.

Frente à falta de evidências na literatura sobre os efeitos da ventilação não invasiva sobre os mecanismos de defesa pulmonar e sobre o conforto em pacientes terminais hipoxêmicos que aceitam uma forma alternativa de suporte de vida sem os prejuízos de uma intubação traqueal, justifica-se a realização deste estudo. 


\section{OBJETIVOS}




\section{2- OBJETIVOS}

O objetivo deste estudo foi observar o efeito da ventilação mecânica não invasiva com máscaras faciais, utilizando-se geradores de fluxo e ventilador microprocessado, em pacientes oncológicos com insuficiência respiratória a fim de verificar:

- Impacto nas propriedades viscoelásticas do muco respiratório;

- Conforto respiratório. 
CASUÍSTICA E MÉTODO 


\section{3- CASUÍSTICA E MÉTODO}

Este trabalho foi realizado no período aproximado de 24 meses. $\mathrm{O}$ local da pesquisa foi a U. T. I. adulto do Centro de Tratamento e Pesquisa Hospital A. C. Camargo (Hospital do Câncer), São Paulo - SP. O estudo do muco respiratório foi realizado no Laboratório de Poluição Atmosférica Divisão de Defesa Pulmonar, na Faculdade de Medicina da Universidade de São Paulo - FMUSP. Todo paciente admitido no estudo, ou seu familiar mais próximo, manifestou aprovação por escrito assinando um termo de consentimento (ANEXO A) no qual foi explicado em linguagem leiga os procedimentos e possíveis riscos e benefícios do protocolo.

\subsection{Sujeitos}

Para a inclusão dos pacientes no estudo foram aceitos os pacientes:

- internados nas UTIs com diagnóstico confirmado de câncer (ou em investigação);

- de ambos os sexos;

- maiores de 18 anos;

- com nível de consciência, que permitisse entender os comandos verbais, ou que necessitasse de baixas doses de sedação e analgesia; 
- todos os pacientes que tinham prescrição médica de Fisioterapia Respiratória e ventilação mecânica não invasiva;

- com diagnóstico de insuficiência respiratória, podendo vir a ser este o motivo da internação ou que viessem a desenvolvê-la durante a hospitalização.

O diagnóstico da insuficiência respiratória (Schettino et al., 2000), será baseado nos seguintes critérios (pelo menos três):

1. Desconforto respiratório com dispnéia ou taquipnéia ( $\mathrm{f}>25 \mathrm{rpm}$ ) ao repouso;

2. Uso de musculatura acessória e ou tiragem intercostal;

3. Gasometria arterial: $\mathrm{pH}<7,35$; e ou $\mathrm{PaO}_{2}<60 \mathrm{mmHg}$ e $\mathrm{PaCO}_{2}>45 \mathrm{mmHg}$

4. Oximetria de pulso: $\mathrm{SpO}_{2}<85 \%$;

5. Padrão radiológico sugestivo de congestão pulmonar.

Critérios para a exclusão do paciente no estudo:

- Instabilidade hemodinâmica: PAS $<80 \mathrm{mmHg}$ e PAD $<60 \mathrm{mmHg}$;

- Parada respiratória;

- Choque, infarto agudo do miocárdio;

- Sangramento digestivo alto;

- Risco de broncoaspiração e incapacidade de manter vias aéreas pérveas;

- Pós-operatório recente de cirurgia esofágica ou gástrica; 
- Pós-operatório ou trauma/deformidade ou queimadura/ferimento facial;

- Pneumotórax não drenado.

\subsection{Materiais}

- ventilador mecânico SAVINA (Dräger Medical AG \& Co. Lübeck Germany, Dräger Indústria e Comércio Ltda, Brasil);

- geradores de fluxo (Vital Signs Inc., Totowa, EUA. Newmed Importadora Ltda, Brasil);

- traquéia de silicone (unindo o gerador à máscara);

- máscara facial de silicone; (Whisperflow Cpap System Gerador Caradyne; Respironics Ireland limited Respironics Inc. - EUA);

- conectores intermediários;

- válvula de PEEP regulável (Vital Signs Inc - Eua. Newmed Importadora Ltda, Brasil);

- circuito do ventilador;

- válvulas redutoras de pressão;

- oxímetro de pulso (AM 78100B Vital Line, ANAMED, Brasil);

- filtros umidificadores (HME) (Make Line Comercial Ltda Me, Brasil);

- monitor cardíaco (DX - 920, Dixtal, Brasil; HP M1205A Viridia Series Monitor, Hewlett - Packard Company, USA);

- Monitor de ECG, Oximetria e pressão não invasiva AM 78100B Vital Line, ANAMED, (Brasil); 
- escova citológica para coleta de secreção nasal;

- lâminas de vidro e estilete para verificação do angulo do muco;

- caixa de isopor para conservação do muco;

- freezer;

- óleo de vaselina e éter de petróleo;

- ringer, água destilada e solução salina ( $\mathrm{NaCl} 0,61 \%)$;

- nebulizador ultra-sônico;

- frascos tipo ependorf de coleta do muco;

- gelo para conservação do muco durante transporte até seu armazenamento.

\subsection{Desenho}

Estudo observacional prospectivo do tipo coorte.

\subsection{Protocolo}

A VNI foi implementada pela equipe de fisioterapia, após diagnóstico de IRp e prescrição de fisioterapia e ventilação mecânica não invasiva no prontuário do paciente, sendo que um grupo usou CPAP com gerador de fluxo e o outro PSV + PEEP.

Para admissão do paciente no estudo foi realizado (ANEXO B):

- diagnóstico de IRp de acordo com os critérios já mencionados.

- avaliação do estado geral contendo descrição do nível de consciência, quadro hemodinâmico (pressão arterial - PA e freqüência cardíaca - FC, uso ou não de drogas vasoativas - DVA) e quadro 
respiratório (freqüência respiratória - f, padrão respiratório, ritmo, uso de musculatura. acessória e ou tiragens intercostal e abdominal), a saturação de oxigênio $\left(\mathrm{SpO}_{2}\right)$ e a $\mathrm{FC}$ foram continuamente monitoradas, através de oxímetro de pulso e monitor cardíaco respectivamente. Foram realizados ainda radiografia de tórax e coleta de gasometria arterial na admissão do protocolo e de rotina. Foram colhidos em alguns pacientes lactato e albumina sérica do dia da admissão;

- dados epidemiológicos: idade, comorbidades, índice de severidade da doença (SAPS II) (Schellongowski et al., 2004), uso de terapia com corticóides inalatórios e endovenosos, antibióticos, antiagregantes plaquetários e trombolíticos, analgesia e sedação, dieta e sondas naso enterais ou gástricas;

- Características da doença: tipo de câncer, data do diagnóstico, estadiamento da doença (TNM), tratamentos específicos realizados previamente ou atualmente e suas intensificações e variações (quimioterapia, radioterapia, cirurgias), TMO, valores de hemoglobina e hematócrito, presença de neutropenia definida como leucopenia < $1000 \mathrm{cel} / \mathrm{mm}^{3}$ e para pacientes com leucemia aguda $<500 \mathrm{cel} . / \mathrm{mm}^{3}$, contagem de plaquetas.

\subsubsection{Fluxograma}




\section{FLUXOGRAMA}

IRp

$\downarrow$

Avaliação/Conforto/Secreção/Gasometria

$\downarrow$

VNI

$\downarrow$

Avaliação/Conforto/Secreção/Gasometria

$\downarrow$

Tratamento a critério da UTI

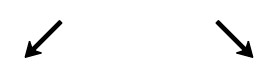

IOT alta 
Todo o procedimento sobre a ventilação não invasiva foi explicado ao paciente antes de mantê-lo por uma hora com a máscara, quando então era reavaliado. Até a colocação da máscara, o paciente permanecia com o mesmo nível de oxigenioterapia que se encontrava no início da avaliação, ou se em ar ambiente, foi submetido à nebulização contínua, com fluxo que permitisse $\mathrm{SpO}_{2} \geq 90 \%$, até a colocação da máscara de CPAP.

O muco nasal foi colhido antes de iniciar o tratamento com os dois sistemas de VNI e uma hora após o uso, a fim de se verificar o efeito agudo dos dois sistemas através da análise das propriedades viscoelásticas.

A coleta do muco foi adaptada de Oliveira (2005) com escova citológica estéril (escova de broncoscopia) introduzida na narina esquerda (ou na direita se houvesse presença de sonda nasoenteral) e feita suave raspagem da região a $6 \mathrm{~cm}$ da abertura nasal externa por poucos segundos.

A seguir a secreção foi retirada gentilmente com uma agulha e colocada em ependorfs contendo vaselina líquida, os quais foram transportados em isopor com gelo e a seguir armazenados sob congelamento em freezer a $70 \cdot \mathrm{C}$ negativos, para análise posterior.

Após uma hora de uso da VNI, foram colhidos novamente o muco, os parâmetros clínicos e o nível de conforto respiratório. A segunda gasometria geralmente foi colhida na rotina da UTI a fim de evitar transtorno desnecessário ao paciente, principalmente aos plaquetopênicos.

A hora de colocação e retirada da máscara de VNI foi registrada exatamente, descontado o tempo de adaptação do paciente ao sistema, quando a fisioterapeuta ficava ao lado dele assistindo e monitorando o 
paciente. Nesse momento também foram ajustados os parâmetros do ventilador.

A PSV foi ajustada a fim de manter volume corrente de aproximadamente 8 mililitros por quilograma de peso corporal ou pressão de pico $<25 \mathrm{cmH}_{2} \mathrm{O}$ e uma freqüência respiratória (f) de 16 a 22 respirações por minuto. Os valores de PEEP foram ajustados do menor valor que possibilitasse $\mathrm{SpO}_{2}>92 \%$ e $\mathrm{FiO}_{2}<60 \%$ variando de 6 a $10 \mathrm{cmH}_{2} \mathrm{O}$, com $\mathrm{FiO}_{2}$ variando de $25 \%$ a $60 \%$ a fim de manter saturação de oxigênio pela oximetria de pulso acima de 92\% (II Consenso de Ventilação Mecânica, 2000). O valor de fluxo deste aparelho neste estudo variou de 80 a 130 litros por minuto.

O grupo do gerador de fluxo tinha apenas o valor de PEEP ajustado através de válvula regulável variando de 5 a $10 \mathrm{cmH}_{2} \mathrm{O}$ e o fluxo de ar do gerador variando de 80 a 100 litros por minuto. $\mathrm{A} \mathrm{FiO}$ podia variar de $33 \%$ a 100\%. Todos estes parâmetros foram ajustados conforme a monitorização respiratória dada pelos valores de gasometria arterial, quando disponível, e pela oximetria de pulso e monitor cardíaco continuamente a fim de verificar a eficácia (ou falência) dos sistemas em reverter a IRp.

Se durante a primeira hora não houvesse melhora da $\mathrm{SpO}_{2}>92 \%$ ou houvesse piora da dispnéia com f > $30 \mathrm{rpm}$, ou o paciente desenvolvesse hipersecreção pulmonar que necessitasse de aspiração nasotraqueal intermitente, o paciente era reavaliado e ficava a critério médico continuar com a VNI ou realizar intubação traqueal, já que estes pacientes podem ter seu estado geral piorado rapidamente (Hill et al., 2007). 
Os pacientes foram intubados, sempre a critério médico, se desenvolvessem instabilidade hemodinâmica severa, PAS $<70 \mathrm{mmHg}$, ou evidência de isquemia ao eletrocardiograma, ou arritmias ventriculares clinicamente significativas e se houvesse diminuição do $\mathrm{pH}<7,25$ (Hill et al.,2007). Doses de sedação (morfina ou benzodiazepínicos) foram aceitas desde que não causassem depressão respiratória.

O uso da VNI foi considerado como sucesso, se a intubação fosse evitada e ocorresse melhora clínica e gasométrica por um período maior que 24 horas, após o término da terapia, ou ainda se apresentasse melhora clínica e com $\mathrm{SpO}_{2}>92 \%$ com baixos fluxos de oxigenioterapia nos períodos sem a VNI.

\subsection{Análise do conforto}

Os pacientes envolvidos no estudo foram avaliados através de uma escala analógico-visual sobre o grau de conforto antes e após o uso do equipamento de VNI (anexo C) e incentivados a referir os achados associados à permanência no mesmo, como: ressecamento de mucosas (nariz e boca), dor no osso nasal, vazamento excessivo e distensão gástrica.

\subsection{Análise do Muco Respiratório}

Neste estudo foram analisadas as propriedades viscoelásticas e de transportabilidade do muco respiratório através dos métodos descritos a seguir. 
Análise in vitro do transporte ciliar do muco respiratório em palato de rã (figura 2):

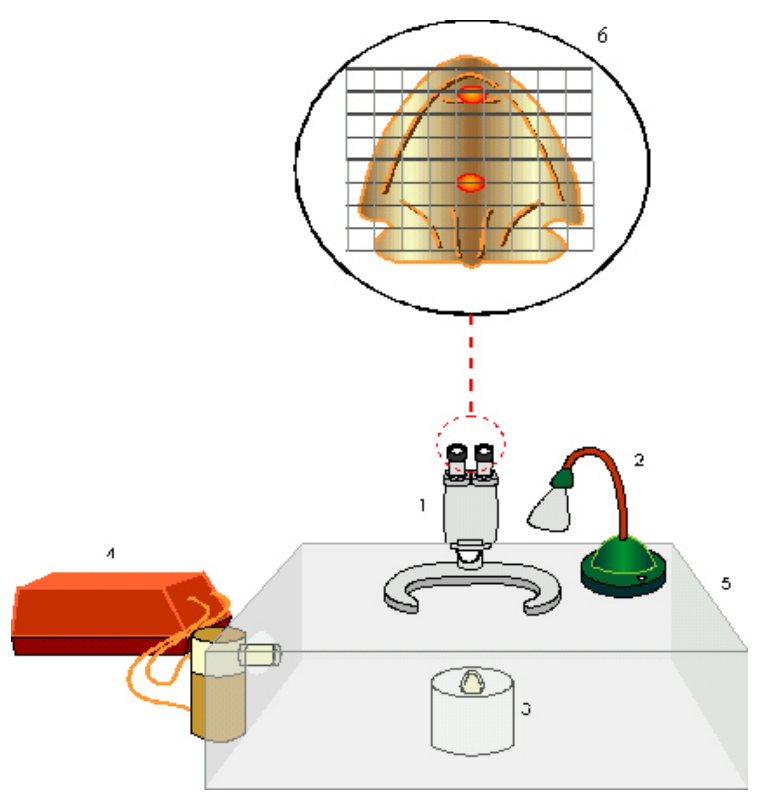

FIGURA 2. Representação esquemática do transporte mucociliar em palato de rã. 1- lupa estereoscópica com objetiva e ocular reticulada; 2 fonte de luz externa; 3 - suporte de vidro para o palato de rã; 4 - nebulizador ultra-sônico; 5 - câmara de acrílico; 6 - palato de rã. (cortesia Dra. Naomi Kondo Nakagawa, 2007).

O transporte do muco respiratório pelos cílios foi realizado in vitro, utilizando-se o palato de rã, o qual possui um epitélio semelhante aos das vias aéreas dos vertebrados, facilitando assim, o estudo do transporte do muco.

Rana catesbiana maduras foram imersas em gelo, para anestesia, e decapitadas a seguir, com posterior dissecção da região do palato. O palato foi então mantido em câmara com uma mistura de ringuer e água destilada na proporção $1: 1$, por 48 horas a $4^{\circ} \mathrm{C}$, para que o muco de rã fosse esgotado pela ação de seu próprio aparelho ciliar. Este muco foi então coletado para 
servir como controle, em relação ao muco a ser testado. Durante os procedimentos o palato de rã foi mantido à temperatura ambiente, dentro de uma câmara de acrílico, em ambiente com 100\% de umidade, garantido por uma nebulização ultra-sônica de solução salina a 2/3 diluída em água $(0,61 \%$ de $\mathrm{NaCl})$. A velocidade de transporte ciliar foi aferida pela colocação de uma amostra de muco (cerca de $5 \mu$ l) sobre o epitélio ciliado do palato de rã.

O deslocamento era visualizado através de uma lupa estereoscópica equipada com uma lente reticulada. Foi utilizado um microscópio (Zeiss) com ocular de aumento de 10 vezes e uma objetiva com aumento de 0,8 . Uma das oculares é reticulada, através da qual era possível medir o deslocamento do muco da parte anterior para a parte posterior do palato (6mm), e seu tempo cronometrado.

O resultado final foi expresso em termos relativos, comparando-se a velocidade do muco teste com a velocidade do muco da própria rã (vel rel).

Análise do muco respiratório através do ângulo de contato:

O aparelho de mensuração do ângulo de contato (Figura 3) é formado por uma lupa com braço articulado para movê-la no sentido lateral, para frente e para trás. A lupa tem capacidade de aumento de 25 vezes e sua ocular possui um goniômetro com escala de zero a 180 graus. 


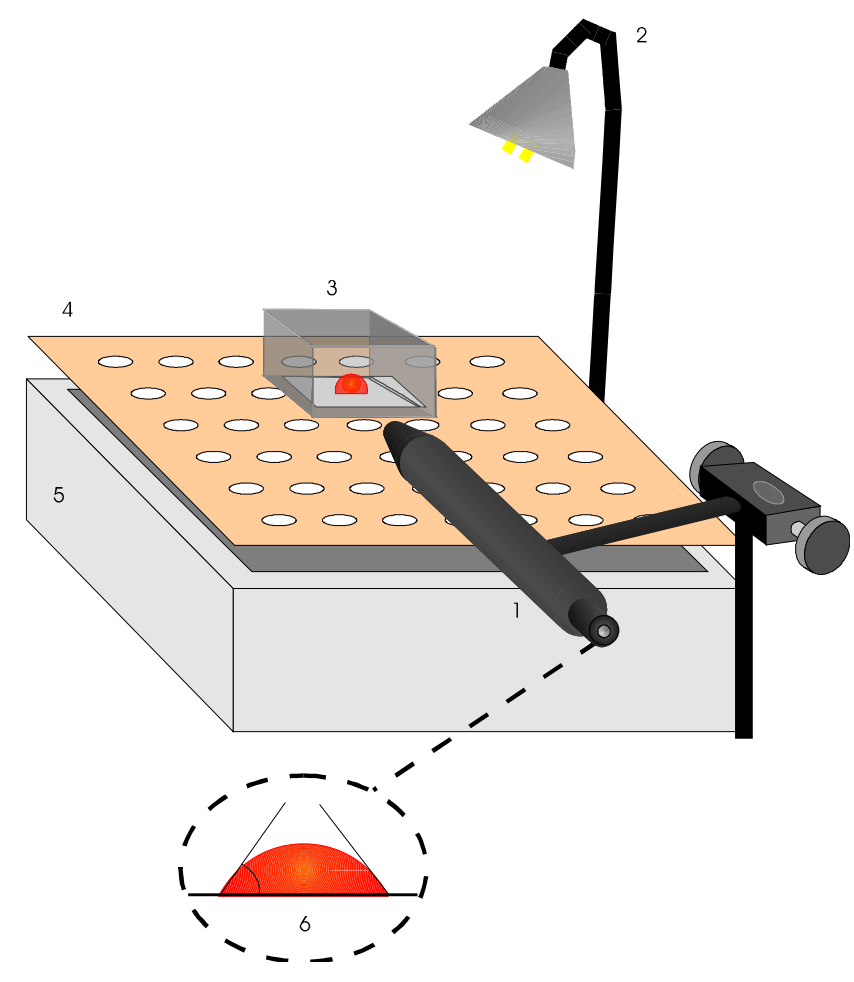

FIGURA 3: Representação esquemática do equipamento de mensuração do ângulo de contato. 1 - lupa com goniômetro e braço articulado; 2 - fonte de luz externa; 3 - câmara de acrílico; 4 - suporte de ferro perfurado; 5 - reservatório de água em banho-maria; 6 - amostra de muco e medida do ângulo de contato. (cortesia Dra. Naomi Kondo Nakagawa, 2007).

Para a mensuração do ângulo de contato, a lâmina foi tratada com solução sulfocrômica para retirada de cargas elétricas da superfície do material que é lavado com água deionizada. As mensurações foram feitas da seguinte forma: banhava-se uma pequena amostra de muco de aproximadamente $5 \mu \mathrm{l}$ em éter de petróleo, e colocada sobre a lâmina tratada, com ajuda de uma agulha. Posicionava-se a lâmina sobre um suporte de ferro temperado, com furos, e este por sua vez em banho-maria à temperatura de $37^{\circ} \mathrm{C}$. 
A amostra de muco fica protegida com uma pequena câmara de acrílico para impedir a sua desidratação por exposição ao ar ambiente e à luz artificial.

A mensuração foi efetuada utilizando-se o goniômetro e foi obtida a medida do ângulo formado entre a gota de muco e a superfície da lâmina.

Análise in vitro do transporte do muco respiratório através de simulador de tosse (Figura 4):

Para a avaliação in vitro do transporte do muco por meio da tosse, empregou-se um simulador de tosse adaptado de King* (1987) citado por Yagi (2002). Este simulador consiste de um cilindro de ar sintético de 49,5 litros, onde sobre pressão de 40 polegadas/libra, o gás é enviado a um solenóide que, por sua vez, oclui o ar em intervalos de dois segundos e se mantém aberto durante meio segundo. O ar é então, transmitido a um tubo de acrílico de $4 \mathrm{~mm}$ de diâmetro interno por $133 \mathrm{~mm}$ de comprimento. O fluxo aéreo obtido é de aproximadamente 235litros/minuto. O transporte do muco através da tosse foi determinado da seguinte forma: uma pequena quantidade de amostra de muco respiratório (de aproximadamente $5 \mu \mathrm{l}$ ) foi banhada em éter de petróleo para remoção do óleo de vaselina. A amostra foi posicionada com um estilete no tubo plástico, onde se efetuou a tosse artificial.

\footnotetext{
${ }^{*}$ King M. Role of mucus viscoelasticity in clearance by cough. Eur J Respir Dis. 1987; 71: 165-172.
} 
O deslocamento do muco foi observado e medido através de uma régua milimetrada.

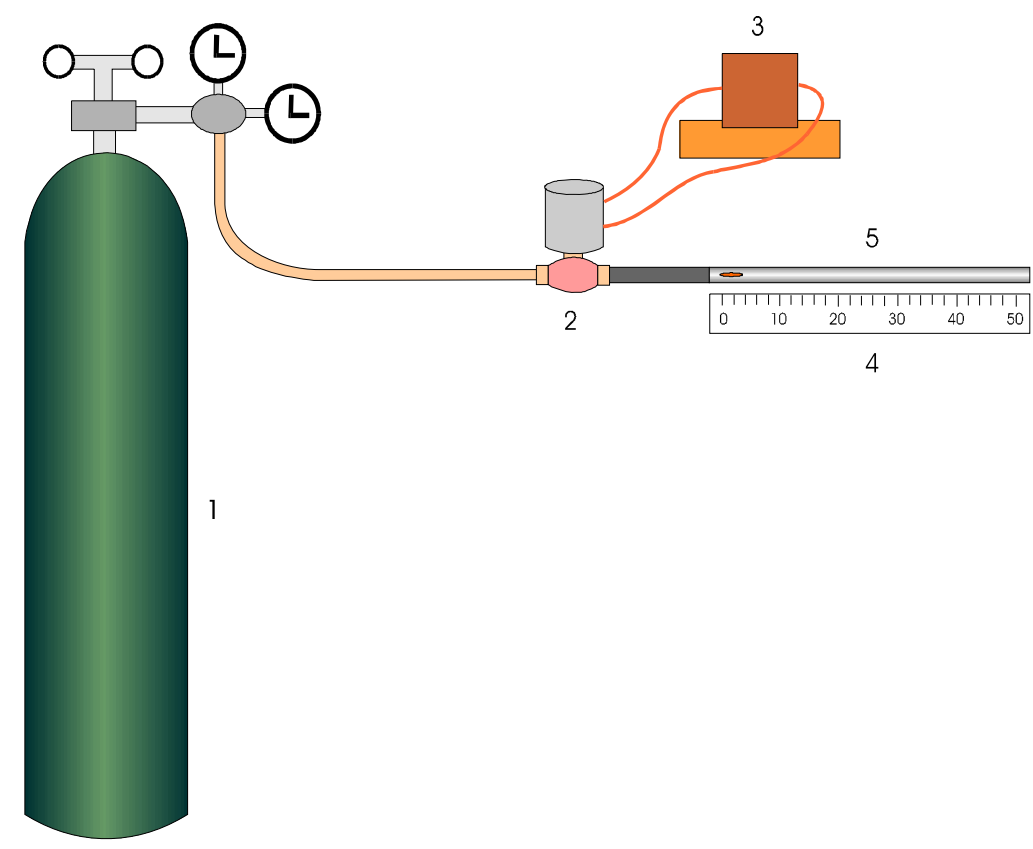

FIGURA 4: Representação esquemática do modelo utilizado para a análise da transportabilidade do muco pela tosse. 1 - cilindro de ar sintético; 2 válvula solenóide; 3 controlador da válvula solenóide; 4 - régua milimetrada; 5 - tubo de acrílico. (cortesia Dra. Naomi Kondo Nakagawa, 2007).

\subsection{Variáveis de estudo}

- Grupos: grupo I (CPAP) e grupo II (PSV+PEEP);

- Características demográficas (sexo e idade);

- Características clínicas: tipo de câncer, tempo desde o diagnóstico, estadiamento da doença, tratamentos específicos, TMO, presença de neutropenia; 
- Escore de gravidade da doença SAPS II (considerado grave acima de 40 pontos, variando de 0 a 147)

- Características reológicas do muco: as propriedades viscoelásticas e de transportabilidade do muco respiratório foram avaliadas através de 3 variáveis: transporte ciliar do muco em palato de rã, análise através do angulo de contato, e transporte através do simulador da tosse;

- Conforto proporcionado ao paciente: medido conforme escala visual (ANEXO C), em três categorias: confortável, indiferente e desconfortável;

- Gasometria arterial;

- Desfecho: alta UTI e IOT/óbito (após terapia com CPAP)

\subsection{Análise estatística}

A análise estatística foi feita utilizando-se o programa SPSS for Windows 10.

Os dados foram testados quanto às características de sua distribuição. Após análise, todos os dados dos dois grupos mostraram-se com distribuição normal. Sendo assim, os parâmetros analisados antes e depois da intervenção foram submetidos ao teste $t$ de Student pareado.

Para análise da diferença estatística entre os grupos, uma vez que a distribuição dos dados era normal, utilizamos o teste $t$ de Student para amostras independentes. 
O nível de significância estabelecido foi de $5 \%(p<0,05)$

Os grupos foram comparados em relação às características demográficas, clínicas, escores, escala de conforto, gasometria e propriedades físicas do muco respiratório, utilizando teste de associação pelo Qui-quadrado (variáveis qualitativas) ou teste t-Student de diferença de médias (variáveis quantitativas).

Para avaliar quais as variáveis estão associadas ao fracasso dos sistemas, o uso da intubação orotraqueal será considerado variável dependente $(\operatorname{sim}=1$ e não $=0)$ e as demais, as variáveis independentes. Será feito o teste de associação pelo qui-quadrado e a seguir, a análise múltipla através da regressão logística.

\subsection{Questões éticas}

Este estudo teve aprovação do Comitê de Ética Hospitalar Centro de Tratamento e Pesquisa - Hospital A. C. Camargo e da Comissão de Ética para análise de Projetos de Pesquisa do Hospital da Clínicas da FMUSP CAPPesq.

Inicialmente houve a intenção de aleatorizar os pacientes nestes dois grupos de tratamento, mas como ainda não há consenso na literatura se é ético realizar estudos clínicos randomizados em pacientes terminais, realizou-se um estudo observacional (Schettino, 2005).

Além disso, este experimento tratou-se de comprovação científica de prática rotineiramente empregada pela fisioterapia no ambiente hospitalar, 
não expondo o paciente a riscos adicionais por participar deste estudo. A coleta de muco foi feita pela fisioterapeuta pesquisadora principal ou por equipe treinada, sempre respeitando o estado geral dos pacientes. Não houve gasto adicional tanto ao hospital quanto aos pacientes, pois os recursos já estavam disponíveis e previstos nos setores.

Os dados obtidos nesta pesquisa serão divulgados em revistas especializadas da área de saúde e mesmo assim a identidade dos pacientes permanecerá sob sigilo absoluto, pois dados como o nome e registro hospitalar não serão divulgados. 
RESULTADOS 


\section{4- RESULTADOS}

\section{1- SUJEITOS}

Foram incluídos dezenove pacientes admitidos na UTI adulto do Hospital do Câncer com diagnóstico de IRp, entre janeiro de 2003 e janeiro de 2005. Do total, quatro pacientes não tiveram amostra de muco nasal suficiente para análise das propriedades físicas do muco e apenas foram excluídos desta análise. Os pacientes recebiam PSV + PEEP ou CPAP de acordo com a disponibilidade dos sistemas no local, ou se já vinham fazendo uso de VNI nas unidades de internação.

Os testes de normalidade mostram que os dois grupos eram semelhantes entre si em relação às características demográficas e clínicas.

Dos dezenove pacientes, dez receberam VNI com aparelho microprocessado (PSV + PEEP) e nove receberam VNI com gerador de fluxo (CPAP).

A distribuição entre os grupos foi bastante homogênea não havendo diferenças estatisticamente significativas entre eles.

As características dos pacientes estão contidas nas tabelas 1 e 2 .

O comprometimento pulmonar é caracterizado por presença de metástase pulmonar, ou segundo tumor primário de pulmão (paciente 12), ou ainda presença de empiema ou derrame pleural. O índice de gravidade dos pacientes utilizado neste estudo foi o SAPS II e teve média de 58,0 pontos e desvio padrão de 11,7 pontos, sem diferença estatística entre os grupos. 
Tabela 1 - Distribuição dos pacientes segundo idade, sexo e acometimento pelo câncer por número de acometidos/total e ou média e desvio padrão(DP).

\begin{tabular}{lcc}
\hline Característica & PSV + PEEP & PEEP \\
\hline & & \\
Idade (anos) - média \pm DP & $60,6 \pm 18,9$ & $70,2 \pm 11,5$ \\
mulheres - n/total & $6 / 10$ & $7 / 9$ \\
tu sólido - n/total & $8 / 10$ & $9 / 9$ \\
tu avançado - n/total & $7 / 10$ & $9 / 9$ \\
sem tumor - n/total & $1 / 10$ & $1 / 9$ \\
comprometimento pulmonar - n/total & $7 / 10$ & $4 / 9$ \\
\hline
\end{tabular}

apical ou paradoxal com tiragem de musculatura intercostal ou de fúrcula; n/total = número de acometidos/ número total de pacientes no grupo

Tabela 2 - Distribuição dos pacientes segundo índice de gravidade, sinais vitais e hipoxemia por número de acometidos/total e ou média e desvio padrão(DP).

\begin{tabular}{lcc}
\hline Característica & PSV + PEEP & PEEP \\
\hline & & \\
SAPS II - média +/- DP & $60,3+/-10,0$ & $56+/-13,0$ \\
abumina sérica média +/- DP & $2,4+/-0,8$ & $2,6+/-0,6$ \\
$\mathrm{f}(\mathrm{rpm})$ - média +/- DP & $32+/-5,7$ & $29,1+/-3,7$ \\
PAM - média +/- DP & $102,0+/-17,5$ & $104,2+/-10,7$ \\
Padrão respiratório & - n/total & $7 / 9$ \\
PaO & /FIO \\
& $9 / 10$ & - média +/- DP \\
apical ou paradoxal com tiragem de musculatura intercostal ou de fúrcula; & \\
n/total = número de acometidos/ número total de pacientes no grupo &
\end{tabular}




\subsection{CAUSAS DA INSUFICIÊNCIA RESPIRATÓRIA}

As causas de IRp com a presença de hipoxemia importante $\left(\mathrm{PaO}_{2} / \mathrm{FiO}_{2}<200\right)$ e o desfecho após 24 da intervenção estão descritas na tabela 3.

Tabela 3 - Causas de IRp e desfechos

\begin{tabular}{|c|c|c|c|c|c|}
\hline Pcte & Causa & $\mathrm{VNI}$ & $\begin{array}{c}\mathrm{IRp} \\
\text { hipoxêmica }\end{array}$ & $\begin{array}{l}\text { IOT } \\
\text { Após } \\
24 \mathrm{~h} .\end{array}$ & $\begin{array}{l}\text { Alta } \\
\text { UTI }\end{array}$ \\
\hline 1 & $\mathrm{SDRA}^{*}$ & PSV + PEEP & Sim & Sim & \\
\hline 2 & SDRA & PSV + PEEP & Sim & & Sim \\
\hline 3 & SDRA & CPAP & Sim & & \\
\hline 4 & DPOC DESCOMPENSADO & CPAP & Sim & & Sim \\
\hline 5 & PNEUMONIA/ PÓS EXTUBAÇÃO & CPAP & & & \\
\hline 6 & PNEUMONIA/ SDRA & CPAP & Sim & & \\
\hline 7 & SDRA / IMUNOSSUPRESSÃO & CPAP & Sim & & \\
\hline 8 & EPC & PSV + PEEP & Sim & & Sim \\
\hline 9 & CRISE ASMATICA & PSV + PEEP & Sim & & Sim \\
\hline 10 & DPOC DESCOMPENSADO & CPAP & & & Sim \\
\hline 11 & $\begin{array}{c}\text { PNEUMONIA/ PROGRESSÃO DA } \\
\text { DOENÇA }\end{array}$ & PSV + PEEP & Sim & & Sim \\
\hline 12 & $\begin{array}{c}\text { PNEUMONIA +CHOQUE SÉPTICO } \\
\text { + EMPIEMA }\end{array}$ & PSV + PEEP & & Sim & \\
\hline 13 & SDRA / IMUNOSSUPRESSÃO & PSV +PEEP & Sim & & Sim \\
\hline 14 & PNEUMONIA/ SDRA & CPAP & Sim & & \\
\hline 15 & EPC & CPAP & & & Sim \\
\hline 16 & $\begin{array}{c}\text { PÓS EXTUBAÇÃO/ EMPIEMA/ } \\
\text { CHOQUE SÉPTICO }\end{array}$ & PSV + PEEP & & Sim & \\
\hline 17 & PNEUMONIA ASPIRATIVA & CPAP & & Sim & \\
\hline 18 & $\begin{array}{l}\text { PÓS-OPERATÓRIO CIR. } \\
\text { TORÁCICA/ SDRA }\end{array}$ & PSV + PEEP & Sim & Sim & \\
\hline 19 & BRONQUIECTASIA INFECTADA & CPAP & & & \\
\hline
\end{tabular}

* ver lista de abreviaturas 


\subsection{PROPRIEDADES FÍSICAS DO MUCO}

O sistema microprocessado (PSV + PEEP) recebeu nariz artificial (HME) em oito dos dez pacientes e o grupo do CPAP (gerador de fluxo) não recebeu nenhum tipo de umidificação nesta primeira hora. A temperatura na UTI ficou em média de $20^{\circ} \mathrm{C} \pm 2^{\circ} \mathrm{C}$.

Houve diferença estatística na transportabilidade do muco pelo palato de rã no grupo da PSV+PEEP que usou o nariz artificial, quando os sujeitos foram comparados antes e depois da intervenção $(p=0,04)$. Também houve diferença no ângulo de contato entre os sujeitos do grupo CPAP, (ar frio e seco), antes e após intervenção $(p=0,06)$ (Tabela 4 e gráficos $1,2,3)$.

Tabela 4 - Distribuição dos valores das propriedades físicas do muco segundo ângulo de contato, máquina da tosse e velocidade relativa e o tipo de VNI (valores em média e desvio padrão).

\begin{tabular}{l|cccccc}
\hline \multirow{2}{*}{ Medida } & pré & pós & $p$ & pré & pós & $P$ \\
\hline \multirow{2}{*}{ Ângulo } & $54,34 \pm 16,44$ & $56,34 \pm 11,27$ & 0,78 & $43,89 \pm 19,17$ & $51,00 \pm 14,85$ & 0,06 \\
Tosse & $14,83 \pm 8,49$ & $12,00 \pm 8,51$ & 0,59 & $30,89 \pm 15,15$ & $17,78 \pm 10,22$ & 0,26 \\
Vel rel & $0,60 \pm 0,25$ & $0,88 \pm 0,45$ & $0,04^{*}$ & $0,70 \pm 0,38$ & $0,66 \pm 0,21$ & 0,95 \\
\hline
\end{tabular}




\section{PALATO DE RÃ}

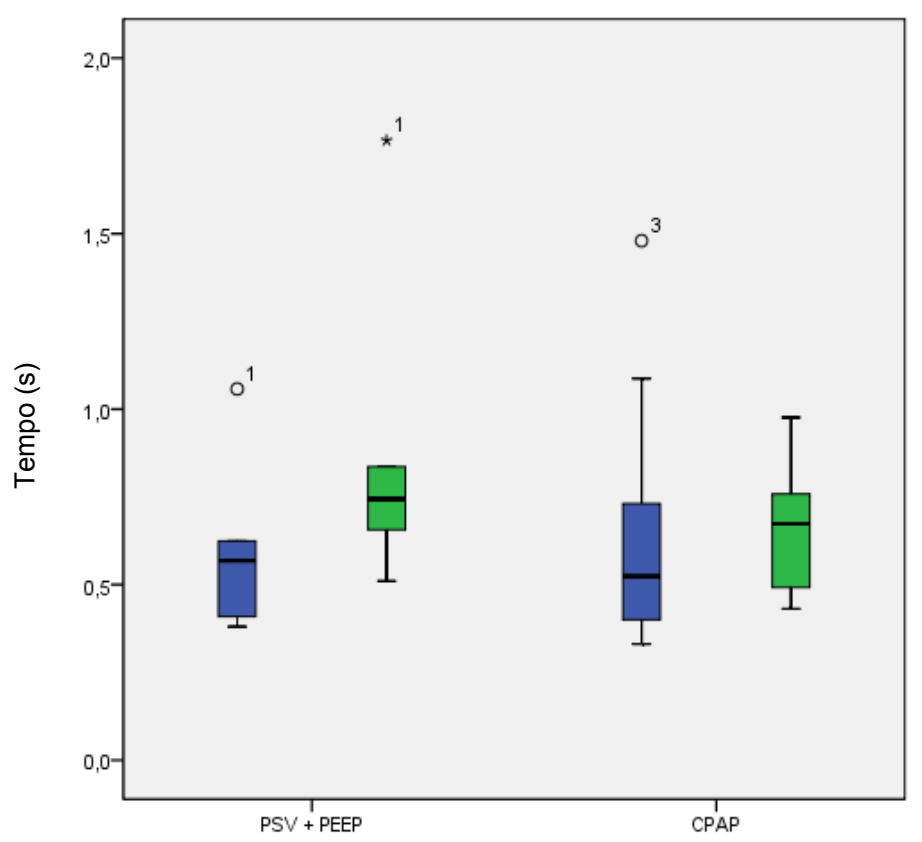

Pré

Pós

Gráfico 1: Box plot mostrando a variação da velocidade relativa do muco no palato de rã. Distribuição dos valores obtidos pré e pós 1 hora de VNI nos dois grupos (PSV + PEEP e CPAP).

\section{MÁQUINA DA TOSSE}

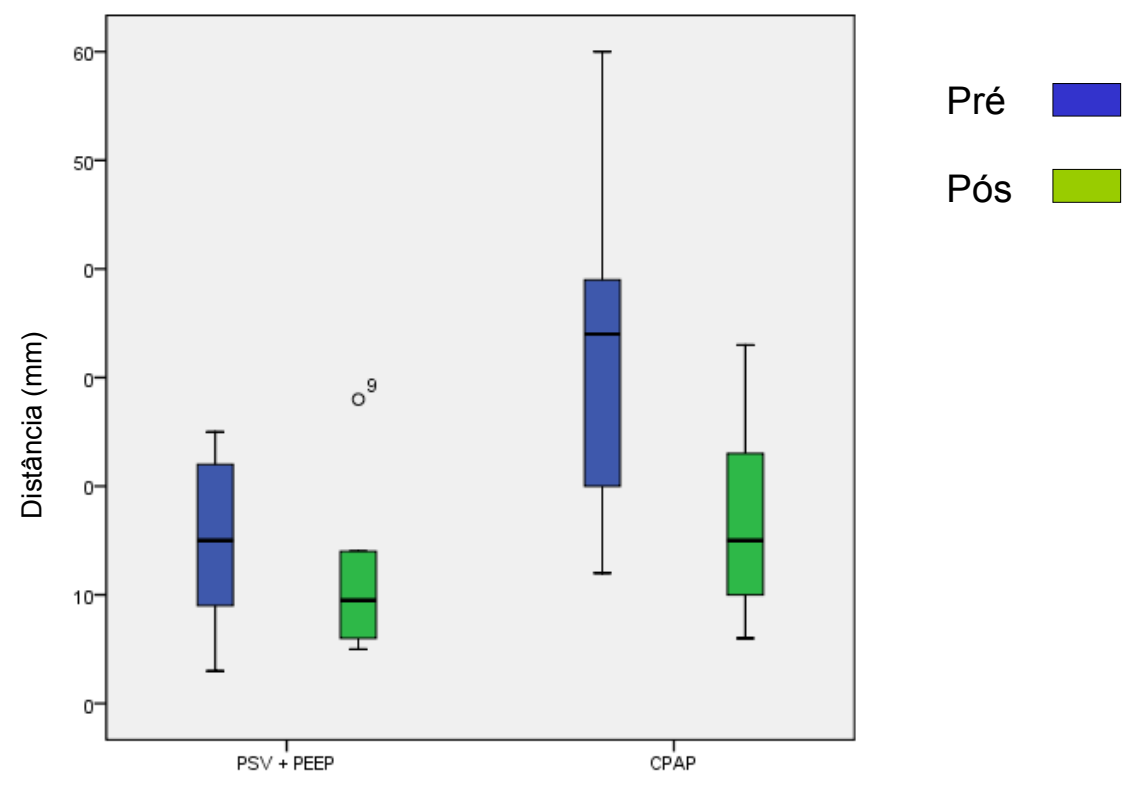

Gráfico 2: Box plot mostrando a variação do transporte in vitro do muco na máquina da tosse. Distribuição dos valores obtidos pré e pós 1 hora de VNI nos dois grupos (PSV + PEEP e CPAP). 


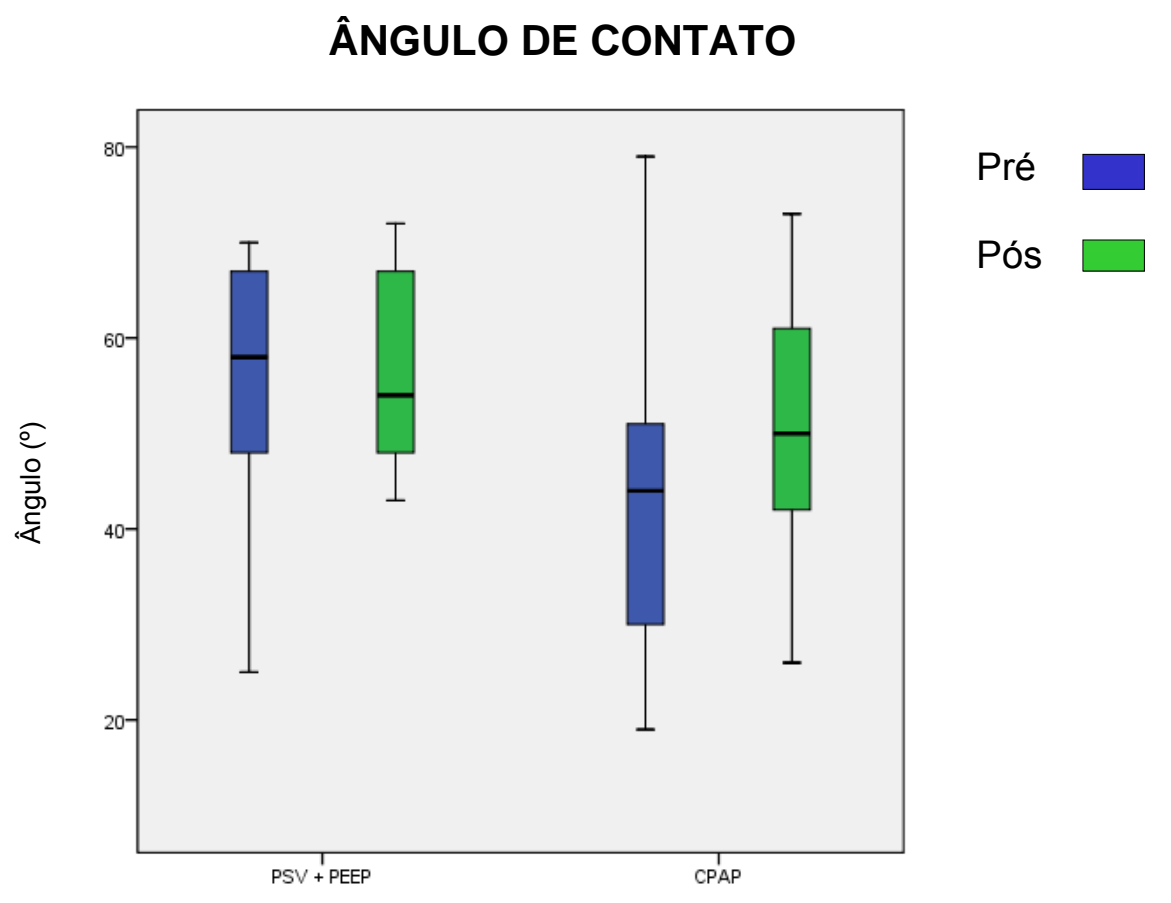

Gráfico 3: Box plot mostrando a variação do muco no ângulo de contato. Distribuição dos valores obtidos pré e pós 1 hora de VNI nos dois grupos (PSV + PEEP e CPAP).

\subsection{PARÂMETROS CLÍNICOS E RELAÇÃO $\mathrm{PaO}_{2} / \mathrm{FiO}_{2}$}

Os dois sistemas de VNI foram eficazes em trazer melhora significativa nos sinais vitais (freqüência respiratória, pressão arterial média, saturação de oxigênio no oxímetro de pulso). Pela gasometria houve melhora na relação $\mathrm{PaO}_{2} / \mathrm{FiO}_{2}$ e na saturação de oxigênio arterial. Também houve melhora no conforto respiratório medido pela escala visual (Tabelas 5 e 6). 
Tabela 5 - Parâmetros clínicos e relação $\mathrm{PaO}_{2} / \mathrm{FiO}_{2}$ pré e pós PSV+PEEP. Valores em média e desvio padrão (para a escala de conforto, só a média foi usada).

\begin{tabular}{lccc}
\hline \multirow{2}{*}{ Parâmetros } & \multicolumn{2}{c}{ PSV + PEEP } & $p$ \\
\hline $\mathrm{f}(\mathrm{rpm})$ & $32 \pm 5,7$ & $23 \pm 5,6$ & 0,006 \\
$\mathrm{PAM}(\mathrm{mmHg})$ & $102,0 \pm 17,5$ & $93 \pm 17,64$ & 0,061 \\
$\mathrm{SpO}_{2}(\%)$ & $87,8 \pm 4,6$ & $97,1 \pm 1,7$ & $<0,001$ \\
$\mathrm{SaO}_{2}(\%)$ & $92,5 \pm 4,9$ & $98,1 \pm 1,0$ & 0,008 \\
$\mathrm{PaO} / \mathrm{FlO} 2$ & $159,6 \pm 79,98$ & $274,9 \pm 87$ & $<0,001$ \\
Escala de conforto & 2,5 & 1,8 & 0,008 \\
\hline
\end{tabular}

Tabela 6 - Parâmetros clínicos e relação $\mathrm{PaO}_{2} / \mathrm{FiO}_{2}$ pré e pós CPAP. Valores em média e desvio padrão (para a escala de conforto, só a média foi usada).

\begin{tabular}{lccc}
\hline Parâmetros & pré & pós & $p$ \\
\hline $\mathrm{f}(\mathrm{rpm})$ & $29,1 \pm 3,7$ & $22,5 \pm 4,14$ & 0,002 \\
$\mathrm{PAM}(\mathrm{mmHg})$ & $104,2 \pm 10,7$ & $94,6 \pm 11,62$ & 0,03 \\
$\mathrm{SpO}_{2}(\%)$ & $88 \pm 3,6$ & $98,2 \pm 1,5$ & $<0,001$ \\
$\mathrm{SaO}_{2}(\%)$ & $92,0 \pm 4,8$ & $98,4 \pm 1,4$ & 0,002 \\
$\mathrm{PaO}_{2} / \mathrm{FIO}$ & & $278,3 \pm 145$ & $<0,001$ \\
escala de conforto & $156,8 \pm 102,5$ & 1,7 & 0,008 \\
\hline
\end{tabular}

Todos os pacientes apresentavam na radiografia torácica na admissão ou pré-admissão na UTI, algum tipo de padrão de infiltrado 
intersticial (geralmente do tipo reticulonodular difuso) ou ainda padrão alveolar (de opacificação).

Outro padrão comumente encontrado nestes pacientes foi o de doença pleural, notado por hipotransparência à radiografia torácica de um dos campos pulmonares, bem como o padrão de colapso (ou atelectasia) geralmente caracterizado por elevação do hemidiafragma do pulmão ipsilateral e deslocamento de fissuras.

A grande maioria dos pacientes apresentava mais de um tipo de padrão radiológico.

\subsection{DESFECHO}

Não houve diferença estatística para tipo de VNI e desfecho (IOT após 24h) $(p=0,09)$.

O seguimento dos pacientes foi feito até o desfecho final da internação.

Tabela 7 - Distribuição dos pacientes por número e porcentagem segundo tipo de VNI e desfecho para intubação orotraqueal ou alta após $24 \mathrm{~h}$

\begin{tabular}{lccc}
\hline & \multicolumn{2}{l}{ DESFECHO } & $24 \mathrm{~h}^{*}$ \\
Grupo & IOT & VNI & Total \\
\hline PSV+PEEP & 4 & 5 & 9 \\
CPAP & 1 & 9 & 10 \\
\hline Total & 5 & 14 & 19 \\
\hline
\end{tabular}

$* p=0,089$ resultado pelo qui quadrado de pearson ajustado pelo teste de 
Tabela 8 - Distribuição dos pacientes por número e porcentagem segundo tipo de VNI e desfecho para óbito ou alta ao final do seguimento de cada paciente.

\begin{tabular}{llll}
\hline \multirow{2}{*}{ Grupo } & \multicolumn{2}{l}{ DESFECHO } & FINAL* \\
& Óbito & Alta & Total \\
\hline PSV+PEEP & 4 & 5 & 9 \\
CPAP & 7 & 3 & 10 \\
\hline Total & 11 & 8 & 19 \\
\hline
\end{tabular}

$* p=0,26$ resultado pelo qui quadrado de pearson ajustado pelo teste de Fisher.

Também não houve diferença estatística para o desfecho final desta internação em relação ao tipo de VNI.

\subsection{COMPROMETIMENTO ONCOLÓGICO}

Em relação ao status oncológico dos sujeitos (tabela 9), apenas 2 dos 19 pacientes que foram admitidos não apresentavam tumor maligno, mas apresentaram IRp e estavam em investigação diagnóstica no momento da admissão do estudo.

Tabela 9 - comprometimento oncológico dos pacientes por número de acometidos sobre o total.

\begin{tabular}{lc} 
Comprometimento & $\mathrm{n}$ acometidos / $\mathrm{n}$ total \\
\hline Câncer avançado & $15 / 19$ \\
Comprometimento pulmonar* & $11 / 19$ \\
Metástase & $14 / 19$ \\
Tratamento atual & $11 / 19$ \\
Politratados $^{* *}$ & $12 / 19$ \\
\hline
\end{tabular}

*Comprometimento pulmonar $=$ segundo tumor primário de pulmão, metástase pulmonar, linfangite carcinomatosa de pulmão, empiema ou derrame pleural de repetição; ${ }^{* *}$ Pacientes politratados: mais de três tratamentos para o câncer (cirurgia,quimioterapia, radioterapia e suas variações). 
Entre os restantes, $15(88,2 \%)$ tinham doença maligna considerada avançada no início do estudo; 11 (65\%) tinham comprometimento pulmonar dado pela presença de metástase pulmonar, ou segundo tumor primário de pulmão (paciente 12), presença de empiema e DPOC grave (paciente 4); 14 (82,4\%) já tinham pelo menos 2 metástases à distância e 12 (70,6\%) já tinham realizado mais de 4 tipos de tratamento para o câncer (pacientes politratados).

\subsection{SINTOMAS RESPIRATÓRIOS}

Os pacientes foram estimulados a dizer quais os sintomas respiratórios que surgiram com o uso da VNI. Este relato foi livre, sem o uso de questionários, e o objetivo foi simplesmente saber qual era o maior incômodo relatado subjetivamente, encontrado durante o uso da VNI e os resultados mais encontrados foram:

- Sufocamento/claustrofobia - 9

- Hiperemia osso nasal - 9

- Máscara apertada - 8

- Vazamento - 6

- Dor osso nasal -4

- Abafamento/calor - 5

- Ressecamento garganta-4

Um paciente referiu distensão gástrica e 10 pacientes negaram qualquer sintoma ou simplesmente a toleravam devido alívio na dispnéia 
com a mesma. Todos os pacientes que referiram "abafamento" ou "calor" com a máscara estavam no sistema de PSV+PEEP.

Esses achados não sofreram tratamento estatístico por ser fruto de livre relato dos pacientes, servindo para nortear o tratamento, no ajuste da máscara e dos parâmetros ventilatórios. 
DISCUSSÃO 


\section{5 - DISCUSSÃO}

O objetivo deste trabalho foi observar o efeito da ventilação não invasiva com pressão positiva contínua nas vias aéreas com máscaras faciais, utilizando-se geradores de fluxo (CPAP) e ventilador microprocessado (PSV+PEEP) em pacientes com insuficiência respiratória por comprometimento oncológico. Em relação às propriedades físicas do muco, houve um aumento da transportabilidade in vitro do muco nasal com o sistema PSV+PEEP $(p=0,04)$ e um aumento no ângulo de contato no grupo CPAP $(p=0,06)$. Os dois sistemas foram eficazes em melhorar significativamente os sinais vitais, a $\mathrm{PaO}_{2} / \mathrm{FiO}_{2}$, o padrão e o conforto respiratório e em evitar a IOT nas primeiras 24 horas $(p<0,05)$.

A escolha dos dois sistemas de VNI foi baseada nos estudos de Delelaux et al. (2000), que comparou o uso de CPAP com gerador de fluxo associado a oxigenioterapia e outro grupo que usou apenas oxigenioterapia em pacientes com insuficiência respiratória hipoxêmica e não hipercápnica. Neste estudo houve uma melhora rápida na $\mathrm{PaO}_{2} / \mathrm{FIO}_{2}$, no grupo CPAP, mas que não evitou a IOT em pacientes com lesão pulmonar aguda.

Este presente estudo, foi ainda embasado nas séries de casos de Meduri et al. (1994) sobre o uso da VNI com PSV em pacientes que recusam a intubação endotraqueal e apresentavam IRp.

Além disso, essa era a prática de atendimento a pacientes com IRp neste serviço: após diagnóstico de IRp, se não houvesse melhora com oxigenioterapia com máscara de nebulização ou cateter nasal, houvesse pelo menos dois critérios de IRp, as indicações e contraindicações do uso de 
VNI fossem respeitados, e houvesse anuência da equipe médica responsável, dava-se início a VNI com geradores de fluxo nas unidades de internação e semi-intensiva ou com aparelhos microprocessados nas UTIs.

Neste estudo devido à alta gravidade destes pacientes cuja necessidade de suporte ventilatório era iminente, a coleta não invasiva de muco pela inalação de solução salina hipertônica como descrito por Yagi (2002) não pode ser utilizada. A aspiração nasotraqueal poderia ainda significar um risco desnecessário aos pacientes sem hipersecreção traqueobrônquica como os imunossuprimidos e plaquetopênicos, por ser uma técnica muito invasiva com riscos inerentes ao procedimento como lesão de epitélio e estímulo vagal. Assim, adaptou-se a coleta de Oliveira (2005) com escova citológica estéril (escova de broncoscopia).

Não foi possível coletar o muco respiratório em todos os pacientes submetidos a VNI, pois muitos pacientes não tinham quantidade de muco suficiente no momento do estudo o que resultou na exclusão de 4 pacientes.

$\mathrm{Na}$ análise das propriedades físicas do muco, o epitélio do palato de rã, apesar de fazer parte do trato digestivo de anfíbios, tem características semelhantes ao epitélio das vias aéreas de mamíferos e tem se mostrado útil no estudo do transporte mucociliar em várias condições (Saldiva, 1990; Lorenzi, 1993; Yagi, 2002; Oliveira, 2005; Trindade et al., 2007).

A máquina simuladora da tosse é usada para verificar as alterações na adesividade do muco pela interação ar-líquido: isto corresponde a força necessária de separação entre o fluido adesivo (muco) e a superfície de 
contato aderente (mucosa) como acontece nas doenças hipersecretivas (Macchione et al., 1995; Trindade et al., 2007).

O ângulo de contato reflete a wettabilidade ou hidrofobicidade do muco sobre a superfície de contato, fornecendo uma medida da tensão superficial do muco sobre o epitélio (Macchione et al., 1995; Trindade et al., 2007).

Os pacientes com IRp receberam VNI com PSV+PEEP, onde foi usado um trocador de umidade e temperatura chamado de HME (heat and moisture exchanger), cujo objetivo na rotina do serviço era o de fornecer barreira protetora contra pneumonia associada ao ventilador. Sabe-se que este sistema além de fornecer barreira biológica, também fornece umidificação e aquecimento não mensuráveis neste estudo, mas estimados devido mudanças encontradas na velocidade relativa do transporte mucociliar pelo palato de rã. Neste grupo houve o relato de "abafamento" ou "calor" com o uso da máscara de VNI.

No estudo de Nakagawa et al. (2000) o HME foi comparado com um sistema de umidificação aquecida tradicional durante a ventilação mecânica invasiva e os efeitos nas propriedades reológicas do muco, no ângulo de contato e no transporte pelo palato de rã foram similares para os dois sistemas, mas houve diminuição da transportabilidade pela máquina da tosse após 72 horas de uso do HME.

Teoricamente, pacientes em VNI com máscara facial, não necessitam de umidificação e aquecimento adicionais, visto que os mecanismos de defesa das vias aéreas estão preservados. Araújo et al. (2000) sugerem que 
a máscara facial mantém o gás expirado saturado, equilibrando o gás inalado seco mantendo assim a integridade e a função normal da mucosa das vias aéreas superiores.

O outro grupo de pacientes usou CPAP com gerador de fluxo, com a entrada do ar unidirecional, próxima ao nariz, e a abertura da válvula de PEEP próxima à boca. Neste sistema não há tempo de saturar o ar inalado com o ar expirado que "vaza" pela válvula de PEEP. Isto explicaria nossos resultados onde ocorreu uma pequena diminuição na transportabilidade do muco in vitro (sem significância estatística) e houve um aumento na wettabilidade ou hidrofobicidade do muco após o uso do gerador.

Reflexos colinérgicos ou liberação local de neuropeptídeos podem ser ativados pelo efeito irritante do ar seco e estimulariam a secreção de fluido aquoso pelas glândulas nasais (Salah et al., 1988) e poucas horas de exposição ao ar frio e seco causam aumento no número de células inflamatórias no pulmão, aumento na permeabilidade vascular e aumento na osmolaridade do fluido extracelular. (Larsson et al., 1998).

Como visto por Seybold et al. (1990) a resposta dos componentes individuais do sistema mucociliar a estímulos fisiológicos e patológicos pode ser divergente e que a direção e magnitude de um componente não prediz a de outro.

A função da mucosa respiratória é dependente tanto da magnitude da umidificação, da temperatura dessa umidificação no gás inspirado, do tempo de exposição da mucosa e da saúde do paciente. No modelo proposto por Williams et al. (1996) qualquer desvio no gás inspirado a partir da 
temperatura corporal e $100 \%$ de umidade relativa, causam mudanças térmicas e na massa de água da mucosa. Diferentes níveis de umidificação e aquecimento causam diferentes níveis de disfunção do epitélio respiratório e conseqüentemente no transporte mucociliar.

Muitas vezes os pacientes que recusam ou que não tem indicação de IOT, permanecem horas seguidas, até mesmo dias (com breves períodos de intervalo) sob a VNI e muitas vezes a umidificação é feita através de inalações. Geralmente estão nas unidades de internação comuns, onde podem ficar com seus familiares em seus momentos finais.

Fora das UTIs, o paciente não pode ser monitorizado tão diretamente pela equipe multiprofissional. Os sintomas respiratórios que poderão aparecer serão então subestimados frente ao benefício da VNI em aliviar a "falta de ar" do paciente. Neste grupo de pacientes, que recebem a VNI por longos períodos ou em pacientes que apresentem sintomas respiratórios, alguma forma de umidificação e aquecimento deveria ser instituída, e sempre verificar se há melhor tolerância da VNI e maior conforto ao paciente.

Como proposto por Sottiaux, (2006) a consistência do muco dos pacientes poderia ser avaliada regularmente a fim de verificar se há adequado condicionamento do ar inalado, ponto crucial para prevenir retenção de secreção e maximizar o funcionamento mucociliar.

As propriedades físicas do muco indiretamente refletem sua habilidade de transporte in vivo, e diretamente influenciam os índices de colonização bacteriana. A mucosa do sistema respiratório funciona 
continuamente, então o epitélio nasal tem a mesma cinética do restante do epitélio respiratório (Button et al., 2008). O uso de técnicas não invasivas é sempre bem vindo especialmente em pacientes oncológicos. O estudo das propriedades físicas do muco nasal pode ajudar a entender o que está acontecendo nas vias aéreas mais inferiores durante a ventilação não invasiva.

Neste presente estudo, além das alterações nas propriedades físicas do muco, os dois sistemas mostraram-se eficazes em reverter a hipoxemia e melhorar os parâmetros clínicos, inclusive com melhora do padrão respiratório e desativação da musculatura acessória em todos os pacientes. Isto era mais do que esperado por ser a VNI a primeira escolha no tratamento da insuficiência respiratória nos dias atuais, tornando-se padrão ouro para o tratamento de causas reversíveis de IRp como DPOC exacerbado e EPC, trazendo melhor prognóstico nestas situações.

Poucos trabalhos como o de Cuomo et al. (2004) mostram a eficácia da $\mathrm{VNI}$ em reverter a IRp em pacientes oncológicos com tumor sólido e avançado, e isto se faz cada vez mais necessário, pois o avanço na terapêutica oncológica vem dando maior sobrevida a estes pacientes e evitar ou reverter a IRp faz parte desta evolução.

A grande incógnita é quando este paciente deixa de ser um paciente em tratamento efetivo para receber um tratamento paliativo e aí, quando se deve iniciar ou descontinuar a VNI.

Nos resultados encontrados por Schettino et al. (2005), num estudo de coorte sobre o uso da VNI em pacientes que não tinham indicação de 
intubação, foi verificado que a VNI poderia ser usada para o alívio da dispnéia ou para permitir ao paciente terminar atividades ou assuntos inacabados no fim de sua vida sem perda de autonomia, mas não era capaz de evitar a morte em pacientes terminais.

O estudo de Curtis et al., em 2007 vem trazer um pouco de entendimento nesta questão. Os autores dividem em três categorias os pacientes que recebem VNI devido IRp: 1) VNI em pacientes sem limitações nos tratamentos de suporte avançado de vida; 2) VNI como suporte de vida enquanto familiares e o paciente decidem adiar ou evitar a IOT e 3) VNI como medida paliativa quando paciente e familiares escolhem evitar medidas avançadas de suporte de vida e recebem apenas medidas de conforto.

Eles ainda sugerem que mais estudos devem ser realizados com os pacientes que recusam a IOT e a ventilação mecânica, e que sumarizar estas três categorias, (e permitir a mobilidade do paciente entre elas) mostra a importância de ouvir e respeitar a preferência e os objetivos do paciente, usar racionalmente a VNI e os leitos de UTI, delinear melhor os parâmetros de sucesso e falência da VNI e quais os lugares mais apropriados para o uso da VNI nas categorias 2 e 3.

Um ponto importante do estudo citado foi mostrar que o sucesso da VNI em pacientes terminais deve ser a melhora dos sintomas e a tolerância da VNI a fim de maximizar o conforto enquanto se minimiza os efeitos dos opióides. A falência é dada quando o paciente não fica mais confortável quando recebe a $\mathrm{VNI}$ ou quando ele quer interromper a VNI, ou ainda 
quando se torna incapaz de se comunicar e aí os cuidados paliativos devem seguir sem a VNI.

Em nosso estudo apenas um paciente que teve alta da UTI foi a óbito no quarto fazendo uso da VNI. Os outros pacientes, ou tiveram a VNI suspensa por reversão da IRP (7 pacientes) e foram de alta da UTI ou entraram em falência e receberam IOT (11 pacientes) e só após esta medida foram considerados "fora de possibilidades terapêuticas", ou "terminais", pela equipe médica responsável.

A sensação de "sufocamento" referida por vários pacientes como claustrofobia devido à máscara, mostra a necessidade de aferição do grau de dispnéia antes e depois de qualquer tipo de intervenção, e questiona-se o uso de sedativos que podem mascarar uma falsa melhora e ainda trazer efeitos colaterais como alteração do transporte mucociliar. Isto não se aplica aos casos de transtornos ansiosos já diagnosticados e que necessitem de medicação (a falta desta medicação poderia aí sim agravar os sintomas de ansiedade sob a forma de dispnéia) e neste estudo apenas dois pacientes vinham fazendo uso de ansiolíticos: um paciente referiu melhora grau 3 para 2 e um não teve melhora (grau 2 antes e depois da VNI).

Em nosso estudo, após a primeira hora, 2 pacientes não toleraram absolutamente a VNI por claustrofobia e incômodo pelo ajuste da máscara e receberam IOT em até $24 \mathrm{~h}$. e também devido piora da IRp.

A escala de conforto escolhida foi baseada no estudo de Pompilio (2000), que usou esta escala em sujeitos normais a fim de entender a assincronia da interação paciente-ventilador. Fácil visualização e evitar 
longos questionários neste momento de urgência clínica foi o principal objetivo ao se usar esta escala no presente estudo. A sua principal limitação foi eventualmente gerar confusão com estado de humor, ou medo do paciente em apontar uma "carinha" triste e "desapontar" familiares ou a equipe.

Outro fator observado é que nos pacientes diagnosticados com depressão, apesar da melhora clínica e gasométrica, não houve mudança significativa no grau de conforto pela escala, mas quando questionados verbalmente, referiram alívio na dispnéia. Conforme estudado por Botega et al. (1995), estados ansiosos podem mascarar ou exacerbar sintomas vegetativos e instrumentos que consigam discriminá-los deverão ser utilizados sempre que possível.

Podemos notar que a insuficiência respiratória do tipo hipoxêmica predominou nos dois grupos, e dado também à alta nota do SAPS II, têm-se uma amostra de pacientes bastante graves condizendo com a prática clínica de uma UTI de um hospital especializado no tratamento de pacientes oncológicos.

O uso de um tipo específico de VNI não impediu a ocorrência de um desfecho desfavorável. Isto infelizmente foi esperado pelo índice de gravidade dos pacientes, mas principalmente pelo grau de acometimento oncológico.

Neste estudo estudo há apenas uma tendência de haver menor número de IOT no grupo do gerador nas primeiras vinte e quatro horas, mas que no seguimento final, neste mesmo grupo, a taxa de IOT aumentou. 
Resultado semelhante encontrado por Delelaux et al. (2000) cuja rápida melhora subjetiva e na relação $\mathrm{PaO}_{2} / \mathrm{FiO}_{2}$ na primeira hora em pacientes que fizeram uso do CPAP, não impediu uma redução nos índices de IOT. Neste estudo, no grupo que recebeu a VNI com PSV + PEEP, as taxas de IOT mantiveram-se inalteradas até o desfecho final dos pacientes.

Os dados sugerem que a elevada taxa de IOT dos pacientes deste estudo se deu pelo desfecho natural destes pacientes pela gravidade da doença de base (câncer avançado) e não ao tipo de suporte ventilatório usado.

Neste estudo, pode-se observar que a VNI alterou as propriedades físicas do muco, deixando este mais transportável pelos cílios quando submetidos a um sistema trocador de calor e umidificação (HME) e com aumento na sua wettabilidade quando submetidos a fluxos de ar frio e seco. Notou-se também que os dois sistemas foram eficazes em trazer melhora clínica e gasométrica significativa após a primeira hora de uso, melhora acompanhada de alívio na dispnéia verificada pelo grau de conforto dado por uma escala visual. O desfecho final observado neste estudo deveu-se provavelmente ao fato da população estudada ser formada por pacientes terminais onde o tipo de VNI não evitaria o óbito e seria usada mais como uma ferramenta nos cuidados paliativos no alívio da dispnéia neste subgrupo de pacientes tão especiais. 
CONCLUSÃO 


\section{6 - CONCLUSÃO}

1- No sistema de VNI com PSV + PEEP houve aumento da transportabilidade do muco no palato de rã e no CPAP com ar frio e seco houve aumento do ângulo de contato (adesividade). Os efeitos da VNI sobre o muco respiratório são heterogêneos e aparentemente dependentes da umidificação e aquecimento do fluxo de ar.

2- O tipo de VNI não evitou a IOT em pacientes terminais portadores de tumor sólido e avançado com IRp hipoxêmica grave, mas agudamente trouxe melhora na relação $\mathrm{PaO}_{2} / \mathrm{FiO}_{2}$, no padrão respiratório e alívio na dispnéia. 


\section{ANEXOS}




\title{
ANEXO A
}

\author{
Informe consciente
}

\section{PROTOCOLO DE VENTILAÇÃO MECÂNICA NÃO INVASIVA}

Eu,

declaro estar ciente de

participar de um protocolo de pesquisa sobre o efeito da ventilação não invasiva com pressão positiva nas vias aéreas, que tem por objetivo comprovar os efeitos benéficos da mesma, garantindo assim uma melhora na qualidade de vida dos pacientes oncológicos. Concordo que sei que este consentimento está sendo expedido exclusivamente para a participação nesta pesquisa, sem possibilidade de extensão da mesma autorização para outros projetos.

Este experimento científico teve a aprovação do Comitê de Ética Hospitalar e expõe à mínimos riscos à saúde dos pacientes, por se tratar de comprovação científica de prática corriqueiramente empregada no dia a dia da fisioterapia respiratória em ambiente hospitalar. 0 nome e o registro de identificação do paciente ficarão sob sigilo absoluto sendo usados em revistas especializadas da área de saúde apenas os dados clínicos encontrados, a fim de divulgar os avanços no tratamento dos pacientes oncológicos.

Aos pacientes oncológicos que desenvolvam insuficiência respiratória durante a internação hospitalar, será oferecido o uso da ventilação mecânica não invasiva com pressão positiva sob a forma de dois sistemas: gerador de fluxo e aparelho microprocessado de ventilação mecânica, através de máscara facial.

O objetivo deste trabalho é verificar qual dos sistemas é mais eficaz em reverter a insuficiência respiratória, sem a necessidade de intubação orotraqueal já que este procedimento leva a um maior risco de pneumonia hospitalar e óbito, principalmente nos pacientes imunodeprimidos. Além disso, será verificado se há diferença entre eles em conforto para o paciente e se ocorrem alterações nas características da muco respiratório após o uso.

Antes e após o uso de uma hora contínua de cada um destes sistemas (o paciente ficará respirando com a máscara por uma hora continuamente após um breve período de adaptação) será solicitado ao paciente identificar através de uma escala analógico-visual qual o grau de conforto em que se encontra,

Antes e após a primeira hora de cada procedimento serão colhidos exames laboratoriais e muco respiratório. Durante todo o procedimento o paciente será continuamente monitorado e sob qualquer sinal de falência do sistema (caso o paciente não se adapte, ou 
caso o sistema não seja capaz de reverter a insuficiência respiratória), ou ainda sob a ordem do médico responsável, o paciente será intubado.

Caso o paciente ou sua família desista a qualquer tempo ou recusem-se a participar deste protocolo todas as condutas rotineiras da UTI serão mantidas e o paciente não sofrerá nenhuma represália por sua decisão.

Qualquer dúvida poderá ser esclarecida entrando em contato com a fisioterapeuta Gabriela Marcon Manfrim CREFITO 3/26646-F pelo telefone 98451313 ou 91239774.

Se o pesquisador principal citado acima, não fornecer as informações/esclarecimentos suficientes, por favor entre em contato com o coordenador do Comitê de Ética do Hospital do Câncer - S.P., pelo telefone 2189-5000, ramais 1113 ou 1117.

Por todo o exposto concordo em participar deste protocolo de pesquisa, conforme abaixo assinado.

NOME DO PACIENTE .

DOCUMENTO DE IDENTIDADE N ${ }^{\circ}$ :

RESPONSÁVEL LEGAL

NATUREZA (grau de parentesco, tutor, curador etc.)

DOCUMENTO DE IDENTIDADE : SEXO: $M(\quad) F(\quad)$

DATA NASCIMENTO.: .....................

$\mathrm{N}^{\circ}$ APTO

ENDEREÇO

CIDADE:

BAIRRO:

TELEFONE: DDD ( ..).

São Paulo, 1 I 
ANEXO B

PROTOCOLO $\mathrm{n}^{\circ}$

CENTRO DE TRATAMENTO E PESQUISA

Nome:

$\mathrm{RGH}$

SAVINA( ) GERADOR ( ) Data __ _ _

Dados clínicos à admissão

Exames laboratoriais

\begin{tabular}{|c|c|c|c|c|c|}
\hline & ANTES & APÓS VNI & & À NTES & APÓS CPAP \\
\hline $\begin{array}{l}\text { NC (escala de } \\
\text { Glasgow) }\end{array}$ & & & $\mathrm{PaO}_{2}$ & & \\
\hline PA & & & $\mathrm{PH}$ & & \\
\hline FC & & & $\mathrm{PaCO}_{2}$ & & \\
\hline FR & & & BIC & & \\
\hline Temp. & & & $\mathrm{BE}$ & & \\
\hline $\begin{array}{l}\text { SatO2 } \\
\end{array}$ & & & $\mathrm{ETCO}_{2}$ & & \\
\hline \begin{tabular}{ll|}
$\begin{array}{l}\text { Respirando } \\
\left(\mathrm{O}_{2} / \text { a.a. }\right):\end{array}$ & em \\
\end{tabular} & & & $\mathrm{SatO}_{2}$ & & \\
\hline Mm acessória & & & Lactato & & \\
\hline \multirow[t]{2}{*}{ Ausculta pulmonar } & & & Leucócitos & & \\
\hline & & & $\mathrm{HB} / \mathrm{HT}$ & & \\
\hline \multirow[t]{2}{*}{ Rx de tórax } & & & Plaquetas & & \\
\hline & & & Albumina & & \\
\hline
\end{tabular}


Dados demográficos/ SAPS II /dados oncol.

\begin{tabular}{|l|l|}
\hline Idade & Agentes vasopressores \\
\hline Sexo & DVAs \\
\hline Tumor & Diálise/ hemodiálise \\
\hline Pcte considerado RHD & Fatores de crescimento hematopoiético \\
\hline SAPS II & Corticoideterapia \\
\hline Data de admissão na UTI/enfermaria & Analgesia/sedação \\
\hline Comorbidades & ATB \\
\hline Tempo desde o diagnóstico (data do diagnóstico) & \\
\hline Estadiamento no diagnóstico & SNG/SNE \\
\hline Estadiamento atual & Dieta \\
\hline Tratamentos realizados & Inalação/puffs \\
\cline { 1 - 2 } Tratamento atual & Complicações da VM \\
\hline Metástases & \\
\hline
\end{tabular}


ANEXO C

COMO VOCÊ ESTÁ RESPIRANDO AGORA?

()

$\odot$

$\because$ 
REFERÊNCIAS BIBLIOGRÁFICAS 


\section{8- REFERÊNCIAS BIBLIOGRÁFICAS}

Ambrosino N. Noninvasive mechanical ventilation acute respiratory failure. Eur Respir J. 1996; 9: 795-807.

Antonelli M, Conti G, Bufi M, Costa MG, Lappa A, Rocco M, et al. Noninvasive ventilation for treatment of acute respiratory failure in patients undergoing solid organ transplantation: a randomized trial. JAMA. 2000; 283 (2): $235-41$.

Antonelli M, Conti G, Moro ML, Esquinas A, Gonzalez-Dias G, Confalonieri $M$, et al. A comparison of noninvasive positive-pressure ventilation and conventional mechanical ventilation in patients with acute respiratory failure. N Engl J Med. 1998; 339: 429-35.

Antonelli M, Conti G, Moro ML, Esquinas A, Gonzalez-Dias G, Confalonieri $M$, et al. Predictors of failure of noninvasive positive pressure ventilation in patients with acute hypoxemic respiratory failure: a multi-center study. Intensive Care Med. 2001; 27: 1718-28.

Araujo MTM, Vieira SB, Vasquez EC, Fleury B. Heated humidification or face mask to prevent upper airway dryness during continuous positive airway pressure therapy. Chest. 2000; 117: 142-7. 
Azoulay E, Alberti C, Bornstain C, Leleu G, Moreau D, Recher C, et al. Improved survival in cancer patients requiring mechanical ventilatory support: Impact of noninvasive mechanical ventilatory support. Crit Care Med. 2001; 29 (3): 519-25.

Bersten AD, Holt AW, Vedig AE, Skowronski GA, Baggoley CJ. Treatment of severe cardiogenic pulmonary edema with continuous positive airway pressure delivered by face mask. N Engl J Med.1991; 325: 1825-30.

Botega NJ, Bio MR, Zomignani MA, Garcia Jr C, Pereira WAB. Transtornos do humor em enfermaria de clínica médica e validação de escala de medida (HAD) de ansiedade e depressão. Rev Saúde Pública. 1995; 29 (5): 355363.

Brandão, CO. A última internação hospitalar dos pacientes que evoluíram a óbito intra-hospitalar: análise dos pacientes do Centro de tratamento e Pesquisa Hospital do Câncer - A C Camargo. [dissertação]. São Paulo: Fundação Antonio Prudente; 2003.

Brochard L, Mancebo J, Wysocki M, Lofaso F, Conti G, Rauss A, et al. Noninvasive ventilation for acute exacerbations of chronic obstructive pulmonary disease. N Engl J Med. 1995; 333 (13): 817-822. 
Brochard L. Noninvasive ventilation for acute respiratory failure. Contempo updates. Linking evidence and experience. JAMA. 2002; 288 (8): 932-5.

Button, B, Boucher, RC. Role of Mechanical Stress in Regulating Airway Surface Hydration and Mucus Clearance Rates, Respiratory Physiology \& Neurobiology (2007), doi:10.1016/j.resp.2008.04.020

Constantinidis J, Knöbber D, Steinhart H, Kuhn J, Iro H. Fine-structural investigations of the effect of nCPAP-mask application on the nasal mucosa. Acta Otolaryngol. 2000; 120: 432 - 37.

Conti G, Marino P, Cogliati A, Dell'Utri D, Lappa A, Rosa G, Gasparetto A. Noninvasive ventilation for the treatment of acute respiratory failure in patients with hematologic malignancies: a pilot study. Intensive Care Med. 1998; 24: 1283-8.

Cuomo A, Delmastro M, Ceriana P, Nava S, Conti G, Antonelli M, lacobone E. Noninvasive mechanical ventilation as a palliative treatment of acute respiratory failure in patients with end-stage solid cancer. Palliat Med. 2004; 18: $602-10$.

Curtis JR, Cook DJ, Sinuff T, White DB, Hill N, Keenan SP, et al. Noninvasive positive pressure ventilation in critical and palliative care 
settings: understanding the goals of therapy. Crit Care Med. 2007; 35 (3): $932-9$

Delelaux C, L'Her E, Alberti C, Mancebo J, Abroug F, Conti G, et al. Treatment of acute hypoxemic nonhypercapnic respiratory insufficiency with continuous positive airway pressure delivered by a face mask: a randomized controlled trial. JAMA. 2000; 284(18): 2352-9.

Fu C, Caruso P, Janaina J, Lucatto J, Schettino GPP, de Souza R, Carvalho, CRR. Comparison of two flow generators with a noninvasive ventilator to deliver continuous positive airway pressure: a test lung study. Intensive Care Med. 2005; 31: 1587-91.

Gregoretti G, Confalonieri M, Navalesi P, Squadrone V, Frigerio P, Beltrame F. Evaluation of patient skin breakdown and comfort with a new face mask for non-invasive ventilation: a multi-center study. Intensive Care Med. 2002; 28: $278-84$.

Groeger JS, White Jr P, Nierman DM, Glassman J, Shi W, Horak D, Price K. Outcome for cancer patients requiring mechanical ventilation. $\mathrm{J}$ Clin Oncol. 1999; 17: 991-7.

Guérin C, Girard R, Chemorin C, De Varax R, Fournier G. Facial mask noninvasive mechanical ventilation reduces the incidence of nosocomial 
pneumonia - A prospective epidemiological survey from a single ICU. Intensive Care Med. 1997; 23: 1024-32.

Hilbert G, Gruson D, Vargas F, Valentino R, Gbikpi-Benissan G, Dupon M, et al. Noninvasive ventilation in immunosuppressed patients with pulmonary infiltrates, fever, and acute respiratory failure. N Engl J Med. 2001; 344 (7): 481-7.

Hill NS, Brenan J, Gaspertad E, Nava S. Noninvasive ventilation in acute respiratory failure. Crit Care Med. 2007;35 (10): 1-6.

Jornal Brasileiro de Pneumologia. II Consenso de Ventilação Mecânica. 2000; 26 (Supl 2); maio.

Jornal Brasileiro de Pneumologia. III Consenso de Ventilação Mecânica. 2007; 33 (Supl 2): S92-105.

Larsson K, Gavhed D, Müller-Suur C, Palmberg L. Inhalation of cold air increases the number of inflammatory cells in the lungs in healthy subjects. Eur Respir J. 1998; 12:825-30.

Levy M, Maged AT, Nelson D, Short K, Senechia A, Vespia J, Hill NS. Outcomes of patients with do not intubate orders treated with noninvasive ventilation. Crit Care Med. 2004; 32 (10): 2002-7. 
Lorenzi, Geraldo $\mathrm{F}^{\circ}$. Efeitos da nebulização de solução salina e $n$ acetilcisteína sobre o muco respiratório de ratos [Tese]. São Paulo: Faculdade de Medicina, Universidade de São Paulo, 1993.

Lorenzi Filho G, Saldiva PHN. Aspectos funcionais do aparelho mucociliar. In: Tavares T. Atualizações em Fisiologia - Respiração. São Paulo: Ed. Cultura Médica. 1991; 86-99.

Love, RR, editor. Manual de Oncologia Clínica. Tradução da União Internacional contra o Câncer. $6^{a}$ edição. São Paulo: Fundação Oncocentro de São Paulo; 1999.

Luedke D, McLaughlin TT, Daughaday C, Luedke S, Harrison B, et al. Mitomycin $\mathrm{C}$ and vindesine associated pulmonary toxicity with variable clinical expression. Cancer. 1985; 55: 542-45.

Macchione M, Guimarães ET, Saldiva PHN, Lorenzi-Filho G. Methods for studying respiratory mucus and mucus clearance. Braz J Med Biol Res. 1995; 28: 1347-55.

Meduri, GU. Noninvasive positive pressure ventilation in patients with acute respiratory failure. Clin Chest Med. 1996; 17(3): 513-53. 
Meduri GU, Fox RC, Abou-Shala N, Leeper KV, Wunderink, RG. Noninvasive mechanical ventilation via face mask in patients with acute respiratory failure who refused endotracheal intubation. Crit Care Med. 1994; 22 (10): 1584 90.

Meert AP, Close L, Hardy M, Berghmans T, Markiewicz E, Sculier JP. Noninvasive ventilation: application to the cancer patient admitted in the intensive care unit. Support Care Cancer. 2003; 11: 56-9.

Mehta, S. e Hill, N. Noninvasive ventilation. Am J Respir Crit Care Med. 2001; 163: 540-77.

Nakagawa NK, Franchini ML, Driusso P, De Oliveira LR, Saldiva PHN, Lorenzi-Filho G. Mucociliary clearance is impaired in acutely ill patients. Chest. 2005; 128: $2772-7$.

Nakagawa NK, Macchione M, Petrolino HMS, Guimarães ET, King M, Saldiva PHN, Lorenzi-Filho G. Effects of a heat and moisture exchanger and a heated humidifier on respiratory mucus in patients undergoing mechanical ventilation. Crit Care Med. 2000; 28 (2): 312-7.

Nava S, Cuomo AM. Acute respiratory failure in cancer patient: the role of noninvasive mechanical ventilation. Crit Rev Onco Hematol. 2004; 51: 91103. 
Ogino S, Nose M, Irifume M, Kikumori H, Igarashi T. Nasal mucociliary clearance in patients with upper and lower respiratory diseases. ORL J Otorhinolaryngol Relat Spec. 1993; 55 (6): 352-5.

Oliveira LR. Efeitos agudos da pressão positiva contínua nasal sobre o aparelho mucociliar de indivíduos sadios: impacto da umidificação e vazamento aéreo [dissertação]. São Paulo: Faculdade de Medicina, Universidade de São Paulo; 2005.

Oliveira LR, Yagi CSA, Figueiredo AC, Saldiva PHN, Lorenzi-Filho G. Shortterm effects of nCPAP on nasal mucociliary clearance and mucus transportability in healthy subjects. Respir Med. 2006; 100: 183-5.

Pastores SM. Acute respiratory failure in critically ill patients with cancer Diagnoses and management. Crit Care Clin.2001; 17 (3).

Pompilio CE. Estudo do padrão respiratório de voluntários sob ventilação com pressão de suporte: influência das rampas de pressurização. [tese]. São Paulo: Faculdade de Medicina, Universidade de São Paulo; 2000.

Robbins S L, Kumar V, Cotran R S. Patologia estrutural e funcional. Rio de Janeiro, Ed. Guanabara Koogan, 1996. 
Salah B Dinh Xuan AT, Fouilladieu JL, Lochart A, Regnard J. Nasal mucociliary transport in healthy subjects is slower when breathing dry air. Eur Respir J.1988; 1: 852-5.

Saldiva, PHN. Aparelho mucociliar: aspectos funcionais e métodos de estudo. J. Pneumol. 1990; 16 (3): 161-170.

Sayit E, Aktogu S, Ertay T, Çapa G, Erkmen G, Özbilek E, et al. Effect of chemotherapy on pulmonary epithelial permeability in lung cancer. Nucl Med Biol. 2000; 27 (6): 581-5.

Scarpinella-Bueno MA, Llarges CM, Isola AM, Holanda MA, Rocha RT, Afonso JE. Uso do suporte ventilatório com pressão positiva contínua em vias aéreas (CPAP) por meio de máscara nasofacial no tratamento da insuficiência respiratória aguda. Rev. Ass. Med. Brasil. 43(3): 180-4, 1997.

Schellongowski P, Benesch M, Lang T, Traunmuller F, Zauner C, Laczika K. Comparison of three severity scores for critically ill cancer patients. Intensive Care Med. 2004; 30: 430-36.

Schettino GPP, Altobelli N, Kacmarek RM. Noninvasive positive pressure ventilation reverses acute respiratory failure in select "do not intubate" patients. Crit Care Med. 2005; 33 (9): 1976-82. 
Schettino GPP, Barbas CSV, Amato MBP, Carvalho CRR. Ventilação nãoinvasiva com pressão positiva. In: Carvalho, CRR. Ventilação Mecânica volume 2. Rio de Janeiro: Atheneu; 2000. p.381-95.

Schwarz MI. The diffuse alveolar hemorrhage syndromes. [cited 2001 Feb 23]. Avaible from: http://www.uptodate.com.

Seybold ZV, Mariassy AT, Stroh D, Kim CS, Gazeroglu H, Wanner A. Mucociliary interaction in vitro: effects of physiological and inflammatory stimuli. J Appl Physiol.1990; 68(4): 1421-6.

Shee CD, Green M. Non-invasive ventilation and palliation: experience in a district general hospital and a review. Palliat Med. 2003; 17: 21-26.

Sottiaux TM, Consequences of under-and over- humidification. Respir Care Clin N Am. 2006; 12(2): 233-52.

Surico G, Muggeo P, Mappa L, Muggeo V, Conti V, Lucarelli A, Novielli C, Rigillo N. Impairment of nasal mucociliary clearance after radiotherapy for childhood head cancer. Head Neck. 2001; 23: 461-6.

Trindade SHK, Mello-Júnior JF, Mion OG, Lorenzi-Filho G, Macchione M, Guimarães ET, Saldiva PHN. Métodos de estudo do transporte mucociliar. Rev Bras Otorrinolaringol. 2007; 73(5): 704-12. 
Varon J, Walsh GL, Fromm RE. Feasibility of noninvasive mechanical ventilation in the treatment of acute respiratory failure in postoperative cancer patients. J Crit Care. 1998; 13 (2): 55-7.

Williams R, Rankin N, Smith T, Galler D, Seakins P. Relationship between the humidity and temperature on inspired gas and the function of the airway mucosa. Crit Care Med. 1996; 24 (11): 1920 - 1929.

Yagi CSA. Estudo das propriedades físicas e de transportabilidade do muco respiratório em humanos, obtidos através de dois métodos: escarro induzido e coleta direta durante anestesia geral [dissertação]. São Paulo: Faculdade de Medicina, Universidade de São Paulo; 2002.

Zayas JG, Godfrey CWM, King, M. Tracheal mucus rheology in patients undergoing diagnostic bronchoscopy: interrelations with smoking and cancer. Am Rev Respir Dis. 1990; 141: 1107-13. 